\title{
Polymeric nanoparticles for optical sensing
}

Francesco Canfarotta*, Michael J. Whitcombe, Sergey A. Piletsky.

AUTHOR ADDRESS: Cranfield Health, Cranfield University, Cranfield, Bedfordshire, MK43 0AL, United Kingdom.

ABSTRACT: Nanotechnology is a powerful tool for use in diagnostic applications. For these purposes a variety of functional nanoparticles containing fluorescent labels, gold and quantum dots at their cores have been produced, with the aim of enhanced sensitivity and multiplexing capabilities. This work will review progress in the application of polymeric nanoparticles in optical diagnostics, both for in vitro and in vivo detection, together with a discussion of their biodistribution and biocompatibility.

KEYWORDS: polymeric nanoparticles, diagnostic applications, optical detection, fluorescence, polymeric coatings, multifunctional nanoparticles, silica.

\section{Table of contents}

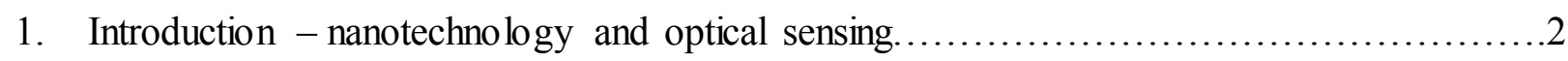

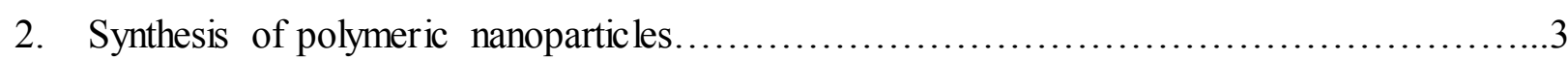

3. Organic polymeric nanoparticles for in vitro optical diagnostics............................5

3.1 Molecularly imprinted polymers...................................................... 10

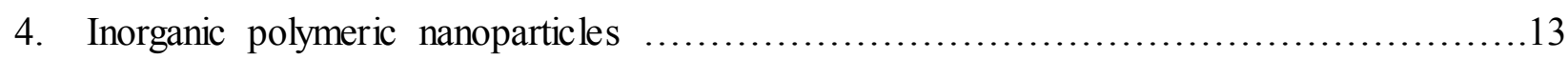

5. Multifunctional polymeric nanoparticles.................................................16

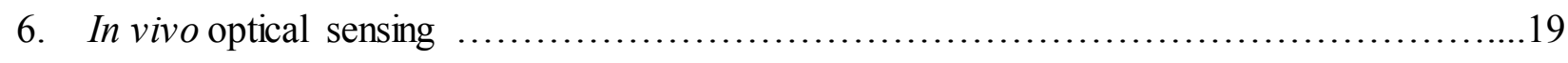

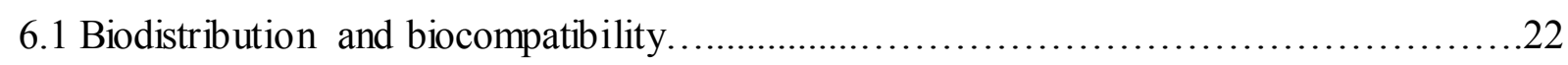

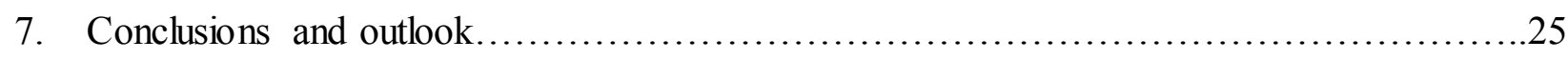




\section{Introduction - nanotechnology and optical diagnostics}

In the past decade, progress in nanotechnology has produced a wide variety of nanoparticles (NPs) useful for diagnostic applications. The use of nanotechnology in diagnostics is attractive because only a small volume of sample is required, allowing a low limit of detection (LOD) to be achieved. Often the use of NPs in diagnostic tests makes them faster to perform and more sensitive than comparable assays which use biomolecules (Jain, 2005). Furthermore, NPs possess unique optical and magnetic properties depending on their composition. Moreover, according to their intended application, nanoparticles can be engineered to impart the required properties. The use of NPs in molecular diagnostics can be termed "nanodiagnostics" and has been successfully employed both in vivo and in vitro assays (Jain, 2007). In recent years, the global market for in vitro diagnostics (IVD) has increased at an incredible rate; from US\$44 Billion in 2010 to a predicted US\$60 Billion by the end 2014 (Renub-Research, 2010). The "point-of-care" sector represents the largest part of the IVD global market, followed by immunochemistry and molecular diagnostics (RNCOS, 2011).

Currently the most important application areas for polymeric NPs in optical diagnostics are biomarker analysis, cancer diagnosis, diagnostic imaging, and immunoassays. The binding of the target biomolecule to NPs is the pivotal step in most nanoparticle-based assays. In order to detect the target analyte, this binding should result in a measurable signal that can be quantified. For this purpose, the most commonly used labels are enzymes, which can catalyze the formation of colored products that can be detected by a change in color of a solution, or molecules/materials capable of emitting a fluorescent signal. Dyes have been popular tools employed in optical diagnostics, allowing the detection of analytes with good sensitivity, either through color changes or by emission of fluorescence. Unfortunately, dyes suffer from photobleaching and often have an asymmetric emission spectrum. Photostability is important in the case of prolonged observation, where photobleaching severely impacts on their capability to detect single molecules. Furthermore some dyes, such as fluoresceins and rhodamines, suffer from quenching phenomena when present in solution at high concentration (Demchenko, 2010). Despite the aforementioned drawbacks, organic dyes are widely employed due to their low cost and ease of use. When the dye is embedded in a polymeric matrix however, increased photostability is often observed due to the "protective" effect of the polymer. Furthermore, covalently coupling the dye to the polymer will also reduce the chance of leakage, which is possible with entrapped dyes, which can still diffuse out of the polymer. NPs can also contain hundreds of dye molecules which will increase the intensity of color or the brightness of emission. Furthermore, the hydrophobic microenvironment created within polymeric NPs can enhance the quantum yield of certain fluorescent reporters (Cui et al, 2013; Hu and Liu, 
2010; Shiraishi et al., 2010; Wang et al., 2009). In addition, the presence of reactive functional groups along the polymer backbone allows specific labeling with other molecules as well as allowing the particle surface to be modified for specific applications.

Reporters for optical sensing can be divided into two main categories. The first includes reporters used as molecular sensors in systems capable of detecting changes in their surroundings and respond to the presence of specific molecules. The second involves reporters used as labels, the aim of which is to give rise to a fluorescent signal which depends only on the presence of the reporter in a particular area of a diagnostic device, without any variation in the fluorescence properties (Demchenko, 2010). The most important considerations when using labels are how to obtain high brightness and provide protection from the surrounding environment. The ideal molecular sensor should possess high molar absorption coefficient and high quantum yield, it should be photochemically and thermally stable, and give an optical response proportional to the concentration of the target analyte without any interactions with other analytes in the sample. A large Stokes shift is highly desirable for both applications because it allows the fluorescent signal to be detected without problems associated with the overlapping of excitation and emission spectra of the dye (Demchenko, 2005). For in vivo use, reporters must be non-toxic and their biodistribution should be well-known. Herein, we describe recent advances in the synthesis and applications of polymeric NPs in optical in vitro and in vivo diagnostics.

\section{Synthesis of polymeric nanoparticles}

Polymeric NPs can be made from organic polymers or inorganic materials such as silica. Generally, organic NPs are prepared either by polymerization of monomers or by processing of preformed polymers. In the latter case, the techniques most frequently employed are: solvent evaporation, salting-out, nanoprecipitation, dialysis and supercritical fluid technology (Rao and Geckeler, 2011). Otherwise, polymeric NPs can be produced from monomers by several methods, such as: dispersion, precipitation and interfacial polymerizations. For diagnostic applications, the most commonly employed techniques are emulsion and living free radical polymerization. The former is carried out in water as the dispersion medium, with or without the addition of surfactant, and allows excellent control to be exerted over the size distribution of the NPs produced, including the production of monodisperse emulsions. Similarly, the living free radical polymerization methods permit excellent control over the molecular weight, polydispersity and composition of NPs (Zetterlund et al, 2008). These latter procedures are based on the establishment of dynamic 
equilibria between a small number of growing radicals and a large majority of the dormant species. Even though this method can have some issues related to the colloidal stability of NPs, living polymerization is an excellent technique for the production of functionalized NPs. Moreover, in contrast to conventional free radical polymerizations, living polymerizations do not undergo exothermic autoacceleration, allowing superior control of parameters such as the polymer chain length and particle size.

Silica NPs are generally synthesized either in an emulsion-based technique performed in organic solvents or by the Stöber method. The first process requires large amounts of surfactant, whereas the Stöber method is performed in mixture of water and ethanol in the absence of surfactants and allows spherical NPs to be obtained in a narrow size distribution. It was recently shown however that the silica layer present on NPs produced by the Stöber method can be inhomogeneous (Wong et al., 2011). In both approaches for the synthesis of NPs, dye can be added to the monomer mixture prior to initiating the polymerization, with the results that it becomes entrapped within the polymeric matrix. Copolymerization with a polymerizable form of the dye can eliminate the leaching of dye from the particle, but generally has a requirement for the chemical structure of the dye to be modified. It has been demonstrated that the incorporation of some dyes can be enhanced by the use of surfactants. However, surfactant can be difficult to remove completely, and its presence could negatively affect the behavior of the particles in biological systems. Silica can be doped with fluorophores by physical adsorption of the dyes. This method however, has several drawbacks such as poor incorporation of the dyes in silica, leakage and aggregation of the dyes, with consequent loss of fluorescence intensity and also variability in the number of dye molecules embedded in each nanoparticle.

Table 1

\section{Organic polymeric nanoparticles for in vitro optical diagnostics}

Regardless of the NP composition, both organic and inorganic NPs can act as signal transducers, transforming the presence of the analyte into a quantifiable optical response caused by changes in photophysical characteristics of the fluorophore. It is worth mentioning that some general requirements for dyes are often mutually exclusive: for instance, high quantum yield might be correlated with a small Stokes shift, or high molar extinction coefficients $(\varepsilon)$ correlate with short fluorescence lifetimes (Demchenko, 2005). Depending on the photochemical process involved in optical detection, we can distinguish three processes important for reporting: (1) generation of fluorescence emission, (2) modulation of fluorescence emission, (3) Förster resonance energy transfer (FRET) processes ( $\mathrm{Li}$ and Liu, 2012). In the first mechanism, fluorescence emission is 
generated by means of specific chemical reactions or recognition phenomena in the presence of the target analyte. For instance, the fluorescence emission of Rhodamine B (RhB) changes according to whether it is in the spirolactam or the acyclic form (Hu et al., 2010; Y. K. Yang, 2005). Hu and colleagues used this phenomenon in the development of RhB-based fluorogenic polymeric micelles for $\mathrm{Hg}^{2+}$ detection. A thermo-responsive hydrophilic block copolymer containing $\mathrm{RhB}$ moieties was prepared using reversible addition-fragmentation chain-transfer (RAFT) polymerization technique. The ring-opening reaction of $\mathrm{RhB}$ moieties occurs after the addition of $\mathrm{Hg}^{2+}$ ions, producing highly intense emission of fluorescence (Hu et al., 2010). In a similar approach a polyethylenglycol (PEG)based thermo-responsive diblock copolymer containing the coumarin moiety was used for the detection of fluoride (Figure 1) (Jiang et al, 2011). Similarly, polymeric micelles based on $\mathrm{Eu}(\mathrm{III})$ complexes and fluorescein isothiocyanate (FITC) were successfully developed. These nanosystems with integrated functionalities showed multicolor fluorescent emission, acting both as temperature and $\mathrm{pH}$ sensors (Li et al., 2009). Wang and colleagues made dual fluorescent polymeric micelles for temperature and $\mathrm{Hg}^{2+}$ sensing able to respond to $\mathrm{Hg}^{2+}$ ions inside living cells with a LOD of $10 \mathrm{nM}$ (Wan et al., 2011). Other reporting groups include the spiropyrans which, upon exposure to UV light, undergo heterocyclic ring cleavage to the open chain merocyanine form. The latter has strong absorption in the visible region, whereas the spiropyran (closed form) is colourless (Chen et al., 2008; Zhu et al., 2011; Zhu et al., 2006). This has been used in the development of spiropyranbased nanosystems which act as fluorometric and colorimetric temperature sensors (Wu et al., 2009b).

By means of a fluorogenic "click" reaction, non-fluorescent alkyne- and azide-derivatives can react to form highly fluorescent products (Figure 2). Using this principle, fluorescent polymeric NPs bearing cyanine dyes have been developed for in vivo targeting of tumor angiogenesis (Deshayes et al., 2011). Miki and colleagues synthesized amphiphilic copolymers which were easily conjugated with folate, PEG and indocyanine green by click reaction. The resulting self-assembled nanosystems efficiently accumulated in the vicinity of the tumor, thanks to the synergistic effect of PEG and folate targeting (Miki et al., 2010). Click chemistry is highly selective and can be easy applied to produce multifunctional systems without employing tedious and time-consuming synthetic procedures. Very recently, such a technique has been applied for in vitro imaging of a glioma cell line by using an azido-terminal fluorescein derivative, which was first synthesized and then reacted with a copolymer bearing alkyne groups (Pucci et al., 2013). As reported elsewhere (Lutz and Zarafshani, 2008), this procedure can also be used to functionalize inorganic nanomaterials, such as magnetic and gold NPs (Álvarez-Paino et al., 2013, Su et al, 2013), silica beads or quantum dots (QDs) (Tissandier et al., 2012). The latter are semiconductor nanocrystals, in 
the size-range of 1-10 nm, composed of sulphides, selenides or tellurides of heavy metals (groups II-VI, III-V, or IV-VI of the periodic table) characterized by high photostability, wide absorption spectrum, intense and typically narrow emission spectrum, large Stokes shift and size-tunable emission (Sahoo et al., 2007). Thus, by varying their size and chemical composition, the emission wavelength can be tuned from the UV to the near infrared (NIR) region, allowing simultaneous detection of multicolor QDs by means of a single light source. Furthermore, they show high fluorescence quantum yields between $650-900 \mathrm{~nm}$, which is the best choice for in vivo applications. Unfortunately, they are potentially toxic due to the presence of heavy metals and require coating with polymer to reduce the risk of heavy metal exposure and to improve their water-solubility (Hezinger et al., 2008). Moreover, Evans and Lovell proved that click chemistry is a useful protocol for the conjugation of several functional molecules even in aqueous media, thus allowing a versatile and simple method for the attachment of water-soluble biomolecules under mild conditions (Evans and Lovell, 2009).

Some non-fluorescent dyes in their single-molecule state can undergo self-aggregation or adsorption process onto substrates, turning on their luminescent emission (Hong et al., 2009, 2011; Luo et al, 2001). Tang and colleagues synthesized polymeric N-isopropylacrylamide (NIPAM)tetraphenylethene (TPE) NPs that act as a fluorescent thermometer, revealing temperature variations within the thermal phase transition of the polymer. This is due to the aggregation process triggered by water (a poor solvent for TPE) and heat (Tang et al, 2009).

The second detection principle (modulation of reporter fluorescence) is based on the intrinsic properties of the bound fluorescent reporter and/or on the responsiveness of the polymer matrix. Some examples of fluorescent reporters which undergo changes in their fluorescence spectra in response to environmental factors are fluorescein and benzylselenide-tricarbocyanine (BzSe-Cy). The emission intensity of the former changes in response to $\mathrm{pH}$ due to its transition between monoand di-anionic states (Bryleva et al, 2007), the latter was employed to develop fluorescent polymeric micelles for the detection of peroxynitrite. In the presence of $\mathrm{ONOO}^{-}, \mathrm{BzSe}^{-\mathrm{Cy}}$ undergoes oxidation, with a consequent decrease in fluorescence emission, allowing intracellular imaging of $\mathrm{ONOO}^{-}$in living cells (Tian et al., 2011). Uchiyama and colleagues developed polymeric temperature-sensitive fluorescent nanogels based on polarity-sensitive dyes with benzoxadiazole (BD) motifs (Uchiyama et al, 2003, 2004). The quantum yield of such dyes is enhanced by thermally-induced aggregation caused by a decrease in the polarity of the microenvironment within the copolymers (Figure 3). At low temperature, the polymer swells by absorbing water and the water-sensitive $\mathrm{BD}$ moieties are quenched by the surrounding water molecules. As the temperature is increased, the polymer shrinks, accompanied by the release of 
water molecules, resulting in fluorescence enhancement due to the $\mathrm{BD}$ units. Such nanosystems are applicable for the monitoring of temperature-dependent intracellular events, as demonstrated by studies with COS7 cells, in which environmental $\mathrm{pH}$ and surrounding proteins did not affect the performance of the polymer (Gota et al., 2009). Similarly, dual fluorescent micelles based on tetramethylrhodamine isothiocyanate and hostasol methacrylate have been synthesized, and were shown to be spontaneously taken up by HeLa cells (Li et al., 2011).

Recently, Gao and colleagues produced block copolymers by atom transfer radical polymerization (ATRP) consisting of poly(ethyleneoxide) (PEO) and tertiary amine-containing (TA) segments with enhanced $\mathrm{pH}$ sensitivity. When the $\mathrm{pH}$ is above the $\mathrm{pKa}$ of the TA block, the copolymers selfassemble into micelles with the TA segments in the core, leading to a quenching effect due to homo-FRET mechanisms. As the $\mathrm{pH}$ is increased, the micelles undergo disassembly since the TA segments become positively charged, with a consequent increase in fluorescence emission. These nanosystems showed a rapid response to changes in $\mathrm{pH}(<5 \mathrm{~ms})$ in the range of intracellular $\mathrm{pH}$ (5.0-7.4) (Zhou et al., 2011). The capability of this system to discriminate $\mathrm{pH}$ values of $0.25 \mathrm{pH}$ units is useful for the evaluation of small differences in $\mathrm{pH}$ within intracellular compartments, which can be less than $1 \mathrm{pH}$ unit (Casey et al., 2010). Unfortunately, no long-term cytotoxicity and photostability studies have been performed to show whether these particular nanosystems might be employed to investigate $\mathrm{pH}$ changes related to cellular aging and molecular alteration in cancer. In contrast, FITC-based NPs were coated with dextran to increase their long-term cytocompatibility with the result that no cytotoxic effects were observed over a 22 day period (Hornig et al., 2008). Other polymeric NPs, mainly based on poly(lactic-co-glycolic acid) and PEG, have been synthesized for imaging of cancer tissue over the last years (Vollrath et al., 2013).

Recently, several types of fluorescent nanoparticles have been employed for the detection of biomarkers (Goutayer et al., 2010; Kelly et al., 2008; Tsai et al., 2011; Wu et al., 2008). For this purpose conjugated polyelectrolytes (CPEs) have been employed since their photophysical properties are known to vary upon interaction with proteins (Li and Liu, 2010; Song et al, 2011). A CPE-based assay has been carried out with aptamer-funtionalized NPs for the detection of lysozyme in mixtures with other proteins, resulting in a LOD of about $0.36 \mu \mathrm{g} / \mathrm{mL}$ (Wang et al, 2010b). Polymeric NPs with an embedded gold core have been employed in luminescence-based assays. The fluorescence emission of reporters such as a cationic polyfluorene was quenched by the metallic core, allowing quantification of the analyte at subpicomolar concentrations of NPs (Fan et al., 2003). Rotello and colleagues have made gold NPs embedded in a fluorescent polymeric shell for quantification of proteins and for differentiation between normal cells and cancer cells using this quenching mechanism (Bajaj et al., 2009; You et al., 2007). The innovation behind this nanosystem 
lies in the exploitation of morphological changes that occur on the cell surface, indicative of various disease states. In another example, pyrene dimers having $-\mathrm{O}-\mathrm{Si}-\mathrm{O}-$ or $-\mathrm{O}-\mathrm{Si}-\mathrm{Si}-\mathrm{O}-$ linkages were synthesized and exhibited different excimer/monomer emission due to the presence of fluoride anions (Gai et al., 2012). In particular, pyrene dimers showed characteristic excimer emission (broad peak around 470-480 nm) together with a weak monomer emission (two sharp peaks at 378 and $396 \mathrm{~nm})$. In the presence of fluoride, the $\mathrm{Si}-\mathrm{O}$ bond is cleaved, thus generating pyrene monomer fluorescence emission. Poly(D,L-lactic acid) (PLA) NPs were loaded with the aforementioned probes and their performance as $\mathrm{F}^{-}$sensor was evaluated in living HeLa cells. When these latter were incubated for 2 hours at $37^{\circ} \mathrm{C}$ in the presence of both fluoride $(100 \mu \mathrm{M})$ and NPs, fluorescence emission characteristic of pyrene monomers was detected. Interestingly, no optical changes were detected in the presence of other ions.

In the third detection principle (FRET), the energy of excitation of a "donor" dye is non-radiatively transferred to an "acceptor" through long-range dipole-dipole interactions. Energy transfer between the donor and acceptor depends on the degree of spectral overlap of the donor emission band and the acceptor absorption band. Furthermore, the relative dipole orientation and the distance between the fluorescent reporters are critical, since the FRET process only occurs when the separation between donor and acceptor is from 1 to $10 \mathrm{~nm}$. Considering these distance-dependent properties, FRET is usually employed as technique for investigating phenomena which lead to variations in molecular distances (Demchenko, 2010). If the acceptor dye is non-fluorescent, the energy transfer leads to quenching. Otherwise, if the acceptor is a fluorophore, a longer-wavelength emission occurs according to the acceptor emission. By employing different donor-acceptor combinations, the FRET process can be tuned, leading to an increase in the Stokes shift. By varying the ratio of the donor-acceptor dyes, multicolor detection can also be achieved, as demonstrated by the work of Wang and Tan (Wang and Tan, 2006). Furthermore, several polymeric nanoparticles employing optical detection systems based on FRET have been developed for bioimaging and biosensing purposes. For different nanosystems the fluorescence is either quenched or enhanced upon addition of the target analyte (Hu et al, 2011a, 2011b; Peng et al., 2010). Other nanoparticles have been prepared which show on/off behaviour of the FRET process, dependent on the presence or absence of the substance to be detected (Chen et al., 2009b; Frigoli et al., 2009; Liu et al., 2012; Ma et al., 2010; Zhu et al., 2007). FRET-based polymeric NPs have been used to detect ionic species, demonstrating good sensitivity. For instance, Frigoli and colleagues used a copper-selective ligand, tetraazacyclotetradecane (cyclam) capable of quenching the fluorescence emission of the embedded acceptor dye (pyrromethene 567) upon interaction with copper, achieving a LOD in the nanomolar range (Frigoli, 2009). By exploiting a similar quenching mechanism, Chen and colleagues prepared 
core-shell polymeric NPs for the detection of $\mathrm{Cu}^{2+}$. In their system the poly(methyl methacrylate) (PMMA) core was impregnated with Nile red, while the shell was made of hydrophilic polyethyleneimine (PEI) for high affinity $\mathrm{Cu}^{2+}$ binding. In the presence of $\mathrm{Cu}^{2+}$, complexation of with PEI at the NP surface leads to quenching of fluorescence emission due to FRET from Nile red to the aforementioned $\mathrm{Cu}^{2+} / \mathrm{PEI}$ complexes. This system showed high selectivity towards $\mathrm{Cu}^{2+}$, since almost no fluorescence quenching was detected in presence of other cations (Chen et al., 2009b). Similarly, Ma and colleagues developed a FRET-based nanosensor for the detection of $\mathrm{Fe}^{3+}$ in aqueous media. They synthesized polymeric micelles based on an $\mathrm{Fe}^{3+}$-reactive $\mathrm{RhB}$ derivative, with switchable fluorescence emission upon $\mathrm{Fe}^{3+}$-induced ring-opening reaction. The system showed good sensitivity, but the irreversible nature of the sensing mechanism might limit its practical application (Ma et al., 2010). FRET-based pH nanosensors have been used for intracellular biosensing in living rat kidney cells. After internalization, the particles were accumulated in intracellular organelles, such as the Golgi apparatus and the endoplasmic reticulum. Interestingly, the size of the nanogel was only slightly affected by drastic changes in $\mathrm{pH}$, and showed good sensitivity over the physiological $\mathrm{pH}$ range. However, the response time of this system towards $\mathrm{pH}$ changes was of the order of several seconds (Peng et al., 2010).

\subsection{Molecularly imprinted polymers}

Usually diagnostic systems rely on biological molecules (antibodies, receptors or enzymes) which exhibit poor stability, especially in organic solvents and at extremes of $\mathrm{pH}$ and temperature. Furthermore, these biological systems are expensive and often difficult to immobilize on the supports used in diagnostic assays (Ye and Haupt, 2004). The use of molecularly imprinted polymer (MIP) nanoparticles can overcome these problems. In molecular imprinting, monomers and crosslinkers are polymerized in an appropriate porogenic solvent in the presence of template molecules. After removal of the template, this matrix retains recognition cavities that are complementary to the template, in terms of size, shape and functionality (Figure 4). The synthesized polymer is able to rebind the template or its structural analogues. Compared with antibodies, the synthesis of MIPs is simpler and cheaper. In addition, MIPs show high stability and excellent mechanical properties (Piletska et al., 2009; Svenson and Nicholls, 2001). Therefore, they can be used in both organic and aqueous media, and can be prepared for a wide variety of targets (Piletsky et al., 2006; Poma et al., 2010). Template removal, however, is often difficult and may not be complete, with the potential for subsequent leaching of analyte from the matrix during analytical applications, resulting in inaccurate performance (Lorenzo et al., 2011). Moreover, it is also quite difficult to integrate them with transducers or to convert the template binding into an electric signal (Mayes and Whitcombe, 
2005; Piletsky et al., 2006). Compared to bulk MIPs, the nanosize form has several advantages: it allows the system to exhibit a much higher surface-to-volume ratio and greater total surface area per weight unit of polymer. The imprinted sites are more easily accessible by the templates, thus improving binding kinetics and template removal, and hence enhancing their recognition capabilities (Gao et al, 2007; Tokonami et al., 2009).

The use of biomolecules (antibodies, enzymes, DNA, etc.) in diagnostic assays is widespread and, to date, represents the gold standard in terms of sensitivity and affinity. However, MIPs are gaining more and more success in the diagnostic field, due to continuous improvements in their synthesis and in the recognition and quantification process of the analyte (template). For instance, Yang and colleagues immobilized a fluorescent compound, 1-pyrenemethylamine (PMA) in MIP NPs containing $N, N^{\prime}$-methylene-bis-acrylamide (MBA) as cross-linker, through Michael addition reaction between the residual $\mathrm{C}=\mathrm{C}$ bonds of the cross-linker and the $-\mathrm{NH}_{2}$ of the fluorescent compound (Yang et al., 2009a). By using a distillation precipitation polymerization technique, they synthesized NPs imprinted for $R, S$-propranolol. The obtained NPs were monodisperse (260 nm) and applicable for selective quantification of propranolol in complex samples, thanks to the high specificity of the system. In another example, fluorescent MIP NPs for atrazine were synthesized by Liu and colleagues, who employed a zinc(II) protoporphyrin-derivative as both functional monomer and fluorescent reporter (Liu et al, 2011b). MIP fluorescence was quenched in response to the rebinding of template with a LOD of $1.8 \mu \mathrm{M}$ and the system showed low cross-reactivity.

Over the last years MIPs have been integrated with other nanomaterials such as gold NPs, QDs, silica NPs or upconverting materials to combine the recognition properties of MIPs with the specific properties of the aforementioned nanomaterials (Descalzo et al., 2013; Ge et al., 2011; Kim et al., 2012; Qian et al., 2013). In one such example, QDs have been employed as fluorescent reporters in MIP-based sensors. If QDs are close to the imprinted recognition sites, the analyte binding quenches the photoemission of the QDs. This is due to the Förster resonance energy transfer, which can result in up to a 4-fold reduction in the photoemission of QDs for some analytes (Guan et al., 2008). Recently, composite QD-MIPNPs have been prepared for the detection of target molecules in saliva, which might be used as biomarkers in the diagnosis of pancreatic cancer (Lee et al., 2010b). It is interesting that the size of such MIP NPs was not significantly affected by the incorporation of QDs. The binding of the target biomolecules to the QDs-MIP NPs was detected by a decrease in the fluorescence intensity. The detection limits of this system were around $0.1 \mu \mathrm{g}$ $\mathrm{mL}^{-1}$ for amylase and lipase, and $0.013 \mu \mathrm{g} \mathrm{mL}$ for lysozyme making it suitable for diagnostic applications, considering that the biological concentrations of lipase are around $0.1-10 \mu \mathrm{gL}^{-1}$ and 
0.1-3 mg mL $\mathrm{m}^{-1}$ for amylase. This MIP system was compared with the Architect ci 8200 system, showing an average accuracy for amylase and lipase QD-MIP NPs of $96.21 \pm 2.44 \%$ and 96.15 $\pm 1.10 \%$, respectively (where the $100 \%$ is complete agreement with the Architect analysis system). In a similar example, Lin and colleagues prepared QD-MIP composite NPs for the detection of lysozyme, creatinine and human serum albumin in urine samples, through evaluation of the fluorescence quenching effect (Lin et al., 2009). These NPs were also compared with the commercial ARCHITECT ci 8200 system, showing good average accuracies. However the accuracy of detection by albumin-imprinted QDs-MIP NPs was only 76.1\%, which shows that the proposed imprinting approach requires further improvement. By exploiting the core-shell approach, Lin and colleagues prepared QD-MIP NPs for caffeine detection based on CdSe/ZnS core-shell QDs functionalized with 4-vinylpyridine (Lin et al., 2004). As in previous cases, the template rebinding resulted in a decrease in the fluorescence emission intensity of the QDs, showing also good specificity in the presence of caffeine analogues (theophylline and theobromine). In another example, Diltemiz and colleagues synthesized QDs-MIP NPs imprinted for guanosine (Diltemiz et al., 2008). Methacryloylamidocysteine (MAC) was used to introduce methacryloyl groups onto the surface of $\mathrm{CdS}$ QDs. Afterwards, the methacryloyl-activated QDs were added to a mixture containing the metal-monomer complex: methacryloylamidohistidine-Pt(II), guanosine, ethylenglycol-dimethacrylate (EGDMA) and azoisobutyronitrile (AIBN) as the radical initiator. The metal monomer complex allows for highly selective recognition through strong interactions between $\mathrm{N} 7$ of guanine and $\mathrm{Pt}(\mathrm{II})$. Like QDs in general, these imprinted QDs possess a broad separation between excitation and emission wavelengths (300 nm and $600 \mathrm{~nm}$ respectively). These nanosystems might be used for DNA sensing or in studies of mutagenesis for the diagnosis of DNA errors. Other authors synthesized Mn-doped $\mathrm{ZnS}$ quantum dots with a surface layer of MIP for the optical detection of pentachlorophenol with an LOD of $86 \mathrm{nM}$ in water (He-Fang Wang, 2009). Since the detection method was based on room temperature phosphorescence (RTP) which has longer emission lifetimes, fewer interferences were observed due to autofluorescence and light scattering. Moreover, this system has no need for inducers or for derivatization since the RTP comes directly from the QDs. Similarly, Li and co-authors synthesized CdSe QDs embedded in a MIP matrix for the detection of $\lambda$-cyhalothrin through concentration-dependent fluorescence quenching ( $\mathrm{Li}$ et al, 2010). These synthesized materials exhibited high photostability and showed good recognition properties, even in presence of other contaminants.

A way to increase the sensitivity of the system when using optical detection is to exploit surfaceenhanced Raman spectroscopy (SERS). For this application, composite gold-core MIP-shell NPs, imprinted with $(S)$-propranolol, were synthesized by seeded emulsion polymerization (Bompart et 
al., 2010). The detection sensitivity achieved $(0.1 \mu \mathrm{M})$ was several orders of magnitude higher than for conventional Raman measurements on plain MIP particles. Furthermore, this detection capacity was also preserved in the presence of a 100-fold excess of interfering compounds such as acetylsalicylic acid or caffeine. Very recently, also silver NPs were used to develop a glucose sensor made by in situ synthesis of Ag NPs within a MIP matrix (Wu et al., 2012). The synthetic route employed allowed silver NPs to be confined in close proximity to each other, so that plasmon coupling effect was achievable. In the presence of glucose, swelling/shrinking processes occur, thus changing the interparticle distance between silver NPs present in the MIP matrix. Therefore, color change of the particle dispersion from yellow to red was easily observed by naked eye. In order to achieve good sensitivity and selectivity together with a visible color change, the optimal amount of silver NPs to be loaded in the polymeric matrix is between $40-50 \mathrm{wt} \%$.

\section{Inorganic polymeric nanoparticles}

NPs based on inorganic polymers such as silica have been applied in several biomedical fields due to their versatility, biocompatibility and simple chemical modifications. As mentioned above, the encapsulation of organic dyes in a silica matrix increases their photochemical stability. Zhao and colleagues developed stable tetramethylrhodamine-doped silica nanoparticles (SNPs) linked with a DNA-probe for sandwich DNA assay, which showed increased photostability after prolonged excitation compared to the same free dye. This system showed a detection limit of $0.8 \mathrm{fM}$, with capability to discriminate one-base mismatches in ssDNA targets (Zhao et al, 2003). Photostability is important for prolonged observation, for instance in diagnostic imaging: the biological environment of living cells can cause degradation of organic fluorophores and the problem of photobleaching can compromise the acquisition images from the sample. The use of an inert silica matrix can help to overcome these issues, as demonstrated in the work of Shi and colleagues. They synthesised SNPs with embedded carboxytetramethylrhodamine (TAMRA), using a reverse microemulsion method. Such NPs were selectively accumulated in lysosomes of HeLa cells, and their fluorescence detected over a 5-day postrecultivation period, showing a photostability 30 times higher than the widely used lysosome marker LysoTracker Green. The long-term cell viability was not affected by the presence of such NPs and their lysosome labeling ability was independent of the dye used in the NPs, promising that a variety of similar SNPs could be employed for multicolor labeling inside living cells (Shi et al., 2010).

The monitoring of intracellular $\mathrm{pH}$ conditions is often employed for the evaluation of cellular diseases. In general, membrane-permeant esters of some dyes can be used for $\mathrm{pH}$ evaluation in cells 
(Tsien, 1981). However, these dyes cannot be targeted to individual organelles and often aggregate, invalidating $\mathrm{pH}$ measurements (Clark et al., 1999). By incorporating the indicator dyes in silica NPs these drawbacks can be overcome, since NPs can minimize organelle sequestration and avoid cytotoxic effects due to direct contact with organic fluorophores (Hornig, Biskup, 2008). In another example, $\mathrm{Xu}$ and colleagues developed core-shell silica nanoparticles (60 nm) containing two dyes (FITC and $\mathrm{Ru}(\text { phen })_{3}{ }^{2+}$ ) which were employed as a ratiometric intracellular $\mathrm{pH}$ indicator $(\mathrm{Xu}$ et al., 2011). This method involves the use of two dyes, one $\mathrm{pH}$ sensitive and one $\mathrm{pH}$ insensitive reference dye, embedded in a silica or polymer matrix. The ratio of the emission intensities of the two dyes can be correlated to the $\mathrm{pH}$. Compared to nanosystems containing only the $\mathrm{pH}$-sensitive dye, ratiometric $\mathrm{pH}$ indicators are more reliable, because the ratios in emission intensity is less sensitive to variations in excitation source intensity and changes in experimental conditions. By employing a modified Stöber method, the authors synthesized co-doped SNPs in which the reference dye was located in the core of the SNPs where it was protected by the silica matrix. The $\mathrm{pH}$ sensitive molecules were grafted onto the surface to maximize their interactions with the environment. Such nanosystems produced color changes from red to yellowish-green over the $\mathrm{pH}$ range from 2.0 to 8.0 in the intracellular micro-environment of hepatoma cells (Figure 5). The same synthetic procedure was employed by Doussineau and colleagues for the development of ratiometric $\mathrm{pH}$-nanosensors based on rhodamine-doped SNPs and a naphthalimide derivative as the $\mathrm{pH}$ sensitive dye (Doussineau et al., 2009). The sensitivity achieved in the physiological pH range was good, but further investigations about their intracellular behavior have still to be performed.

Since hundreds of dye molecules can be embedded in one NP, this will enhance the signal recognition, allowing ultrasensitive detection (Wang, 2006; Wang et al., 2010a). However, the amount of dye embedded must be carefully chosen to avoid self-quenching, typically occurring in many organic dyes at high concentrations (Knopp et al., 2009). Two dyes (Cy3- and Cy5) were encapsulated into SNPs and used in a two-color DNA microarray, revealing higher sensitivity, with a detection limit of $1 \mathrm{pM}$ for target DNA and showing more photostable signals compared to the direct use of free cyanine dyes (Zhou, 2004).

Due to their high fluorescence intensity, SNPs have been employed for protein detection at picogram levels. Tris(2',2-bipyridyl)dichlororuthenium (II) hexahydrate-doped SNPs have been prepared by inverse microemulsion polymerization technique, labeled with antibodies against tumour necrosis factor- $\alpha$ (TNF- $\alpha$ ) and used in a fluoroimmunoassay for the detection of TNF- $\alpha$, showing a LOD of $0.1 \mathrm{pg} / \mathrm{mL}$ (Hun and Zhang, 2007). However, physically entrapped dyes might undergo leaching from the matrix. Studies demonstrated that leaching of dye from SNPs amounted 
to $45 \%$ loss of dye over $48 \mathrm{~h}$, due to weak reactions between matrix and the dye molecules (Clark, 1999). By conjugating FITC with (3-aminopropyl)triethoxysilane (APTS), Gao and colleagues synthesized stable fluorescent SNPs which were employed for the quantification of $\gamma$-globulin, with good sensititvity (LOD of $0.04 \mu \mathrm{g} / \mathrm{mL}$ ) and reduced photobleaching (Gao et al., 2008). Protein quantification has also been achieved through the use of PEG-functionalized silica-coated gold NPs, bearing aptamers or antibodies on their surface (Jana and Ying, 2008). These NPs allowed protein detection by the naked-eye, after protein immobilization on nitrocellulose membranes followed by gold or silver enhancement.

The use of SNPs in optical diagnostics is widespread because they possess several advantages. In fact, they are hydrophilic and biocompatible, and poorly attacked by bacteria. Their size and porosity do not change under variations in $\mathrm{pH}$ unlike polymer particles, which can also swell in organic solvent, leading to dye leakage. Moreover, their surface can be easily modified through appropriate chemical derivatization for the attachment of biomolecules (Kurkina and Balasubramanian, 2011). Furthermore, they do not absorb electromagnetic radiation in the wavelength range from 300 to $800 \mathrm{~nm}$. Unfortunately, SNPs possess poorer multiplexing capabilities compared with quantum dots because of overlap in the dye excitation spectra. Nevertheless, some authors tried to develop SNPs with multiplexing capabilities. In a rare successful example three energy transfer fluorophores were encapsulated inside SNPs and their emission spectra tuned, such that they showed different colors at a single excitation wavelength (488 nm) (Wang and Tan, 2006). The authors labeled such NPs with biotin in order to carry out a biotin-avidin binding assay. The obtained biotin-SNPs were used to cover streptavidin-coated microparticles, as shown by SEM (Figure 6a) and confocal microscope images (Figure 6b) which exhibited different colors. For diagnostic purposes, such SNPs can be conjugated with antibodies for recognition of specific antigens.

In order to exploit a similar streptavidin-biotin based assay, Liu and colleagues synthesized silica nanoparticles by the reverse microemulsion method. The particles were made highly fluorescent by covalently coupling the organic dye Alexa Fluor 647 (AF647) to the particles. The surface of the silica was derivatized with (3-mercaptopropyl)trimethoxysilane bearing thiol groups, allowing the subsequent linkage of a maleimide-streptavidin conjugate. The obtained streptavidin-functionalized SNPs specifically recognised biotin-labelled DNA, allowing a LOD of $0.1 \mathrm{pM}$ to be achieved (Liu et al, 2011a). Similarly, terbium-doped SNPs have been developed for the detection of nucleic acids (Chen et al., 2007). They showed enhanced brightness and 1.5-ms fluorescence lifetime which allowed them to be used in time-resolved fluorescence assays, providing 100-fold higher detection sensitivity compared to the same assay based on FITC (Chen, Chi, 2007). Furthermore, SNPs based 
on europium(III) and synthesized through a modified Stöber method were used for glucose detection in human serum samples. Interestingly, the results obtained were in good agreement with the data obtained by the hospital (Yijishan Hospital, Wuhu, China) by means of a clinically employed glucose analyzer (Hitachi 7170, Japan), showing that this nanoplatform is reliable and suitable for practical uses (Gao et al, 2009).

\section{Multifunctional polymeric NPs}

The simultaneous exploitation of several physical properties possessed by NPs can be beneficial for their application in different fields (de Dios and Diaz-Garcia, 2010). Magnetic SNPs can be integrated with optical reporters for simultaneous cellular imaging and bioseparation, both for in vivo and in vitro applications (Corr et al., 2008; Hwang et al., 2010; Kim et al., 2008; SalgueiriñoMaceira et al., 2006). Likewise, paramagnetic iron oxide NPs and QDs can be embedded within the same polymeric matrix (Figure 7). Silica-coated magnetic NPs were also grafted with QDs by means of a metal ion-driven deposition technique, for simultaneous optical and magnetic detection (Corr, Rakovich, 2008). The magnetic moiety within multifunctional NPs can also be employed for hyperthermic cancer treatment, due to its capability to generate heat upon application of an external magnetic field. Cho and colleagues developed nanocomposites by embedding magnetic NPs into a polystyrene matrix, which was functionalized with NIR-emitting QDs (Cho et al., 2010). The system was able to achieve active targeting in vivo, with multimodal imaging and therapeutic characteristics thanks to the inclusion of the chemotherapeutic agent paclitaxel within the matrix (Figure 7). This study represents an excellent example of versatility, since both bimodal imaging diagnosis and therapeutic treatment are achievable. To produce polymeric magnetic-QDs, iron oxide NPs can be used as cores for the growth of CdSe QDs, with further silica coating to achieve biocompatibility and bioconjugation for cell imaging (Selvan et al., 2007).

In another experiment, magnetic NPs were coated with dye through a reactive silane derivative (Figure 8) and conjugated with folic acid. SNPs with magnetic cores and fluorescent shells were used for imaging of cancer tissues due to specific binding of the particles with the folate receptor, which is up-regulated in several human cancers, allowing enhanced tumor targeting capability (Sudimack and Lee, 2000). Very recently, it has been investigated how folic acid binds to its receptors, whose crystal structure was also determined (Chen et al., 2013). These findings can provide a rationale for the design of drugs specifically targeted for the folate receptors. Magnetic- 
fluorescent SNPs have been also used for cell labeling as demonstrated by $\mathrm{Lu}$ and colleagues, even though no active targeting was employed to improve the imaging performance (Lu et al., 2007).

A similar method has been employed by $\mathrm{Lu}$ and colleagues for the preparation of multifunctional NPs based on upconverting materials. Upconversion refers to an optical process in which the absorption of two or more photons results in the emission of a single higher energy photon. The advantage of using these upconverting NPs lies in the fact that they can be excited with NIR light, which can penetrate deep into tissue and show minimal background autofluorescence since blood and tissues are transparent at NIR wavelengths. Erbium and ytterbium co-doped sodium yttrium fluoride were co-precipitated onto magnetic NPs in the presence of EDTA as chelator. A layer of silica was then deposited in order to protect the crystals and allow the covalent coupling with streptavidin ( $\mathrm{Lu}$ et al, 2004). These types of systems have the advantage of emitting fluorescence after excitation with a NIR light source. These particles, capable of binding to biotinylated molecules, were used as single-molecule imaging probes, aided by their high photostability (Wu et al., 2009a).

Another method used to synthesize fluorescent magnetic NPs is the alternate layer-by-layer deposition of negatively and positively-charged polyelectrolytes. These are deposited through the formation of electrostatic interactions, initially with the negatively-charged surface of the magnetic NPs. This allows tuning of the thickness of the polymer layer by controlling the number of layers allowed to accumulate on the particles. By using this approach, negatively charged CdTe QDs were successfully embedded within the polymeric layers (Hong et al., 2004). The resulting composite NPs showed high luminescence and good magnetic properties, providing another potential multimodal diagnostic nanoplatform. The magnetic properties of the aforementioned multifunctional NPs can also be exploited for bioseparation purposes. For instance, Lee and colleagues developed Rhodamine-labeled magnetic-silica NPs which were modified with PEG and administered to cancer cells in culture. These NPs were internalized into living cells, allowing them to be both fluorescently and magnetically labeled. Interestingly, by application of an external magnetic field the labeled cells could also be actively moved (a process named the "magnetic motor effect"), without any apparent acute toxicity (Yoon et al., 2005). Apart from simultaneous cell separation and imaging purposes, this system might find applications in the magnetic delivery of drugs within cells. A similar nanosystem has been employed for cancer cell and tumor imaging. Nanocomposites made from Rhodamine $B$ and iron oxide NPs embedded within a poly(glycidylmethacrylate) (PGMA) matrix demonstrated good biocompatibility in rats, with the ability to be visualized both through MRI and through fluorescence microscopy of tissue sections (Figure 9) (Harrison et al., 2012). Moreover, they remained at the injection site (optic nerve), thus 
opening the possibility for the delivery of therapeutic agents even to injured neurons. Multifunctional NPs can be also employed in anticancer phototherapy. A novel dual-color system has been recently developed for both cancer imaging and therapy by means of the simultaneous production of nitric oxide and singlet oxygen triggered by light (Fraix et al, 2013). The advantage of such a system relies in the synergistic effect of the species generated, since both NO radical and ${ }^{1} \mathrm{O}_{2}$ possess promising anticancer activity, thus improving the efficiency of the cancer therapy.

In the near future, many other combinations are likely to be developed, with the incorporation of functionalities such as enzymatic catalysis and electrochemical properties. By improving the "multifunctionality" of these nanosystems, a wide choice of capabilities is envisioned for the realization of functionalized probes tailored for specific biomedical applications.

\section{In vivo optical sensing}

Nanotechnology offers the unique possibility to produce systems suitable for in vivo applications, ranging from diagnostics to theranostics and therapy. For these applications, however, NPs should be biocompatible and have clear biodistribution and elimination from the organism. Various materials used in NPs such as QDs carry the potential risk of systemic toxicity, in this case due to the presence of heavy metals. A typical solution to this problem is coating the source of toxicity with an inert polymer such as silica. Several reviews describing surface modifications of QDs for in vivo applications have been published recently (Dey et al., 2009; Sperling and Parak, 2010; Xing et al., 2009). Considering their biocompatibility, SNPs have been also employed as carriers for NIR dyes for in vivo imaging of mouse bladder (Burns et al., 2009), and for specific tumor imaging (Benezra et al., 2011). The main factors which affect the acute toxicity of SNPs include shape, porosity and their surface characteristics (Nakamura et al., 2007, Yu et al., 2012). These latter affect also the colloidal stability of nanosystems in vivo, which might agglomerate and determine an immune response. Stabilization against agglomeration can be achieved by using hydrophilic polymers which create a physical barrier between particles, thus reducing sterically the interactions among NPs.

The analytes which require detection or imaging vary from small molecules to cells. For instance, the overproduction of hydrogen peroxide is involved in the progression of several diseases, and agents that can be used to measure hydrogen peroxide in vivo attract great interest. Murthy and colleagues synthesized polymeric micelles containing peroxalate ester groups (Lee et al., 2007). The latter generate a specific intermediate (dioxetanedione) in the presence of $\mathrm{H}_{2} \mathrm{O}_{2}$, which can excite fluorescent dyes through a chemiluminescence mechanism, which allows hydrogen peroxide 
to be detected in organisms such as mice (Lee et al., 2011) (Figure 10). The authors achieved nanomolar sensitivity and good specificity for hydrogen peroxide over other reactive oxygen species. This system was further improved by adding a PEG corona to the particles to evade macrophage phagocytosis and by reducing the NP size down to $33 \mathrm{~nm}$ to enhance extravasation into permeable tissues (Lee et al., 2008).

Multimodal polymeric micelles have been developed for the visualization of cancer cells, known to overexpress a specific receptor, by both optical and nuclear techniques (Figure 11). The accurate information provided by this system might be useful for the early detection of cancer and its angiogenesis (Zhang et al, 2011). To achieve active targeting, specific peptide sequences were conjugated to NPs, increasing the specificity of tumor imaging (Mulder et al., 2009).

Other nanosystems based on nuclear and optical detection exploit near infrared fluorescence (NIRF) imaging which, as mentioned above, allows low tissue absorbance and minimal in vivo autofluorescence to be achieved (Ntziachristos et al., 2003). Thus, Yang and colleagues developed PEG-coated micelles with embedded near-infrared fluorescent dye for dual optical and nuclear imaging applications, showing a prolonged blood residence and effective accumulation inside solid tumors in mice (Figure 12) (Yang et al., 2007).

Both passive targeting, through Enhanced Permeability and Retention (EPR) effect (Decuzzi et al., 2009), and active targeting (Lee et al., 2010a; Liang et al., 2010; Zheng et al., 2012) were exploited in the application of NPs. In the example of passive targeting (EPR effect), Kim and colleagues developed polymeric NPs containing the fluorogenic probe Cy5.5 and the dark quencher BHQ-312, linked together by a peptide sequence specific for a matrix metalloproteinase (MMP) for in vivo tumor imaging (Lee et al., 2009). MMPs are a family of zinc-dependent proteins involved in inflammatory diseases and cancer progression. When these NPs are exposed to the specific MMP, fluorescence emission of Cy5.5 occurs, due to the enzymatic cleavage of the peptide bond between Cy5.5 and the quencher. The specificity of tumor imaging is usually enhanced by the conjugation of NPs with specific antibodies. Other moieties used in specific targeting include folic acid (Hong et al., 2008; Suen and Chau, 2013, Yoo et al., 2012), galactose (Yoo et al., 2007), peptides (Rowe et al., 2009; Zhang et al., 2010) and cell ligands (Chen et al., 2009a).

\subsection{Biodistribution and biocompatibility}

The size of nanoparticles is the parameter which chiefly affects their biodistribution (Riehemann et al., 2009). Typically particles with diameter smaller than $100 \mathrm{~nm}$ are considered for in vivo 
applications, to reduce opsonization and assure subsequent clearance. It is however still unclear how variations in the size of NPs within the range from 20 to $100 \mathrm{~nm}$ may influence their biodistribution (Yang et al, 2009b). The biodistribution of NPs also depends on their surface properties (Stark, 2011). The role of the polymeric coating on circulation lifetime was investigated by Ballu and colleagues who injected PEG-coated QDs into the blood stream of mice. These remained in the blood circulation for an extended period of time (half-life around $3 \mathrm{~h}$ ), whereas organic dyes were eliminated within minutes after their administration. Incredibly, the fluorescence of these nanosystems was detected even after four months in vivo (Ballou et al., 2004). Such features are due to the hydrophilic polymer coating that reduces opsonisation and reticuloendothelial uptake (Ballou et al., 2007). By studying the biodistibution and urinary excretion of three types of surface-modified silica NPs (OH-SNPs, COOH-SNPs, PEG-SNPs), it has been shown that both clearance time and organ deposition are dependent on the surface characteristics of NPs (He et al., 2008).

Once NPs enter in the organism, they undergo several biological processes. Typically, NPs in the bloodstream are opsonized and then sequestered in the reticuloendothelial system (RES), in order to be destroyed, but if they are not biodegraded, NPs may accumulate in cells and tissues with potential toxic effects. Thus long-term biocompatibility studies have to be performed, in order to clarify the potential risk arising from NP accumulation within cells. One approach to reduce cellular internalization and reduce cytotoxicity is to modify the surface of particles with neutral hydrophilic polymers. PEG with $\mathrm{MW}>2000 \mathrm{Da}$ is a particular useful modifying agent for increasing the blood circulation time of NPs, thanks to its ability to reduce the adsorption of opsonins by means of steric repulsion forces (Owens and Peppas, 2006; Yang et al. 2007). In the case of non-PEGylated NPs, the sequestration in the RES is rapid, typically a matter of minutes, with most of the NPs being concentrated in the liver and spleen. Addition of a PEG layer shifts the biodistribution towards the spleen (Owens and Peppas, 2006). To further increase the blood circulation time and reduce cytotoxicity, some authors combined PEG with chitosan in the particle coatings (Sheng et al. , 2009). Interestingly, particles with diameter $>200 \mathrm{~nm}$ show a more rapid clearance than smaller particles, regardless whether they have a PEG coating or not. In general, the characteristics of the polymeric coating such as thickness, surface density, charge and functional groups affect the biodistribution of NPs, since they influence the interaction of the particles with opsonins (Owens and Peppas, 2006).

Dextran is another neutral material which is clinically approved for NP coating for in vivo applications (Brigger et al., 2002). Other examples include chitosan, hyaluronic acid (both natural polymers), and poly(acrylic acid) and polyethyleneimine (synthetic polymers). Innovative dextran- 
coated silica NPs showed enhanced biocompatibility since they were able to degrade into renallycleared components, thus reducing the in vivo toxicity in mice (Park et al., 2009). This is the first example of NPs engineered to degrade in vivo into harmless components over specific timescales.

As mentioned above, many authors employ silica coatings to obtain biocompatible NPs. Such fluorescent SNPs were employed for in vivo injection and subsequent histological analysis. Peritoneal cells were analyzed by confocal fluorescence microscopy, which revealed that SNPs were distributed on the surface and inside the cells, far from the nuclei, with no apparent toxicity for up to 1 month (Nakamura et al., 2007).

Generally, the evaluation of toxic effects in based on whether or not NPs are able to determine cell death. More studies are required in order to assess the toxicological effects of NPs, such as alterations in the natural morphology or functions of the cell, prior to their approval for in vivo applications. For QD-based nanosystems (even coated with polymers or silica), the cytotoxicity may arise from the potential release of heavy metals (Lewinski et al., 2008; Selvan et al., 2010). Furthermore, the capping reagents, such as triocytlphosphine oxide (TOPO), employed during their synthesis, could also have cytotoxic effects on biological systems (Weng and Ren, 2006). Small polymer-coated QDs, however, might be eliminated from the body by excretion through the kidney (Gao et al., 2004). Thus, further studies are needed to understand the clearance mechanisms of QDs, before their use in humans.

It is worth mentioning that the potential for cytotoxicity depends mainly on the surface characteristics rather than on the embedded core. However, NPs might not lead to apparent acute in vivo toxicity, but could accumulate within organs. Thus, extensive studies are needed to understand the long-term effects of nanosystems due to their accumulation in organs. Apart from the intrinsic properties of NPs, their toxicity depends also on dose and route of administration (Yildirimer et al., 2011). For instance, recent studies suggested that polymeric NPs around $300 \mathrm{~nm}$ have higher level of intestinal absorption compared to bigger particles (600 and $1000 \mathrm{~nm}$ ), thus giving a general direction in the rational design of NPs for oral drug delivery (He et al., 2012).

When a NP starts its "journey" within the blood stream, it is transported by the flow of blood until it interacts either specifically (through ligand-receptor bonds) or non-specifically (electrostatic, van der Waals and steric interactions) with the blood vessel walls. NPs tend to move differently from the blood vessel core towards the vessel walls, (a process named "margination dynamics") depending on their shape (Ferrari, 2008). Spherical NPs tend to follow the blood flow and move parallel to the vessel walls, whereas discoidal NPs drift laterally from one side of the vessel to the other. Interestingly, if NPs are not driven by active targeting forces, they tend to leave large blood vessel in favor of smaller ones, thus passively accumulating in the microcirculation (Figure 13). 
Lateral drift towards the endothelium is observed only in the presence of an external force (such as a magnetic field) or because of electrostatic and van der Waals interactions with the vessel walls (Decuzzi et al., 2009). Hence, when NPs have to be designed for in vivo applications, their shape and coating must be chosen carefully, to allow a proper biodistribution and accumulation in the target area. Moreover, the size, shape and surface coating of NPs have an important role to play in the intracellular pathways of internalized NPs, as demonstrated by PEG-coated QDs (Duan and Nie, 2007). A recent theoretical model showed that large spherical particles are more susceptible to internalization within cells compared to small and elongated ones (Decuzzi and Ferrari, 2008). Thus, the internalization process could be controlled by tailoring the particle shape. Very recently, the particle uptake into HeLa cells of fluorescent polystyrene NPs having different surface charges and sizes was studied (Lerch et al., 2013). The results proved that positively charged NPs are taken up more efficiently than neutral or anion particles, probably because the positively charged polymeric layer interacts electrostatically with the negatively charged glycocalyx present on the cellular surface. Even the internalization mechanism depends on the size: for particles $<500 \mathrm{~nm}$, receptor-mediated endocytosis is the main pathway; larger particles $(>1 \mu \mathrm{m})$ are internalized through a more complex phagocytic process. Particles between $500 \mathrm{~nm}$ and $1 \mu \mathrm{m}$ undergo internalization through a mixed mode (Decuzzi et al., 2009).

In conclusion, the potentialities of NPs for in vivo diagnostics are evident, and their use is envisaged to grow rapidly in the near future. However, although toxicity studies have been widely performed, definitive protocols for evaluating the toxicology of different types of NPs are still missing. Furthermore, long-term toxicity studies at low concentrations of NPs are urgently required for applications in humans, since diagnostic and therapeutic protocols usually require only small amounts of NPs.

\section{Conclusions and outlook}

Over the past decade the use of polymeric nanoparticles in optical diagnostics has gained momentum, due to progress achieved in the tailoring of their physico-chemical properties. From the reported examples, it is evident that responsive nanosystems and in particular fluorescent nanoparticles have demonstrated great success both for in vivo and in vitro applications. However, more improvement is needed in the controlled synthesis of these materials especially in relation to their reproducibility, size distribution and long-term stability of NP-bioconjugates. Moreover in vivo behavior of NPs must be further examined, in particular with regard to their biodistribution, agglomeration and toxicity. An interesting option for improving the biocompatibility of NPs might 
involve the use of self-destructive components within the NPs, which could be degraded into harmless products after the accomplishment of their purpose in vivo. Regarding the application of nano-MIPs, currently they are not able to replace biomolecules in diagnostic applications, although their potential is evident. Generally a synthesis protocol is still required which enables multifunctional MIP nanoparticles to be produced with a uniform size and shape. It is without doubt, however, that novel responsive nanosystems and innovative platforms with multiplexing capabilities will be developed in coming years for improved optical diagnostic applications.

\section{ACKNOWLEDGMENT}

This research was supported by the Research Executive Agency (REA) of the European Union under Grant Agreement number PITN-GA-2010-264772 (ITN CHEBANA). 


\section{REFERENCES}

Álvarez-Paino M, Marcelo G, Muñoz-Bonilla A, Rodríguez-Hernández J, Fernández-García M. Surface modification of magnetite hybrid particles with carbohydrates and gold nanoparticles via "click" chemistry. Polymer Chemistry. 2013;4:986-95.

Bajaj A, Miranda OR, Kim IB, Phillips RL, Jerry DJ, Bunz UHF, Rotello, VM. Detection and differentiation of normal, cancerous, and metastatic cells using nanoparticle-polymer sensor arrays. Proceedings of the National Academy of Sciences of the United States of America. 2009;106:10912-6.

Ballou B, Ernst LA, Andreko S, Harper T, Fitzpatrick JAJ, Waggoner AS, Bruchez MP. Sentinel lymph node imaging using quantum dots in mouse tumor models. Bioconjugate Chemistry. 2007;18:389-96.

Ballou B, Lagerholm BC, Ernst LA, Bruchez MP, Waggoner AS. Noninvasive Imaging of Quantum Dots in Mice. Bioconjugate Chemistry. 2004;15:79-86.

Benezra M, Penate-Medina O, Zanzonico PB, Schaer D, Ow H, Burns A, DeStanchina E, Longo V, Herz E, Iyer S, Wolchok J, Larson SM, Wiesner U, Bradbury MS. Multimodal silica nanoparticles are effective cancer-targeted probes in a model of human melanoma. The Journal of clinical investigation. 2011;121:2768-80.

Bompart M, De Wilde Y, Haupt K. Chemical nanosensors based on composite molecularly imprinted polymer particles and surface-enhanced Raman scattering. Advanced Materials. 2010;22:2343-8.

Brigger I, Dubernet C, Couvreur P. Nanoparticles in cancer therapy and diagnosis. Advanced drug delivery reviews. 2002;54:631-51.

Bryleva EY, Vodolazkaya NA, McHedlov-Petrossyan NO, Samokhina LV, Matveevskaya NA, Tolmachev AV. Interfacial properties of cetyltrimethylammonium-coated $\mathrm{SiO}_{2}$ nanoparticles in aqueous media as studied by using different indicator dyes. Journal of Colloid and Interface Science. 2007;316:712-22.

Burns AA, Vider J, Ow H, Here E, Oula PM, Baumgart M, Larson SM, Wiesner U, Bradbury M. Fluorescent silica nanoparticles with efficient urinary excretion for nanomedicine. Nano Letters. 2009;9:442-8.

Casey JR, Grinstein S, Orlowski J. Sensors and regulators of intracellular $\mathrm{pH}$. Nature Reviews Molecular Cell Biology. 2010;11:50-61.

Chen C, Ke J, Zhou XE, Yi W, Brunzelle JS, Li J, Yong EL, Xu HE. Structural basis for molecular recognition of folic acid by folate receptors. Nature. 2013.

Chen G, Chen W, Wu Z, Yuan R, Li H, Gao J, Shuai X. MRI-visible polymeric vector bearing CD3 single chain antibody for gene delivery to $\mathrm{T}$ cells for immunosuppression. Biomaterials. 2009a;30:1962-70.

Chen J, Zeng F, Wu S, Chen Q, Tong Z. A core-shell nanoparticle approach to photoreversible fluorescence modulation of a hydrophobic dye in aqueous media. Chemistry - A European Journal. 2008;14:4851-60.

Chen J, Zeng F, Wu S, Su J, Zhao J, Tong Z. A facile approach for cupric ion detection in aqueous media using polyethyleneimine/PMMA core-shell fluorescent nanoparticles. Nanotechnology. 2009b;20.

Chen Y, Chi Y, Wen H, Lu Z. Sensitized luminescent terbium nanoparticles: Preparation and timeresolved fluorescence assay for DNA. Analytical Chemistry. 2007;79:960-5.

Cho HS, Dong Z, Pauletti GM, Zhang J, Xu H, Gu H, Wang L, Ewing RC, Huth C, Wang F, Shi D. Fluorescent, superparamagnetic nanospheres for drug storage, targeting, and imaging: A multifunctional nanocarrier system for cancer diagnosis and treatment. ACS Nano. 2010;4:5398404.

Clark HA, Kopelman R, Tjalkens R, Philbert MA. Optical nanosensors for chemical analysis inside single living cells. 2. Sensors for $\mathrm{pH}$ and calcium and the intracellular application of PEBBLE sensors. Analytical Chemistry. 1999;71:4837-43. 
Corr SA, Rakovich YP, Gun'Ko YK. Multifunctional magnetic-fluorescent nanocomposites for biomedical applications. Nanoscale Research Letters. 2008;3:87-104.

Cui K, Lu X, Guan J, Lu Q, Fei Z, Dyson PJ. Formation and properties of self-assembly-driven fluorescent nanoparticle sensors. Chemistry - A European Journal. 2013;19:8550-7.

de Dios AS, Diaz-Garcia ME. Multifunctional nanoparticles: analytical prospects. Analytica chimica acta. 2010;666:1-22.

Decuzzi P, Ferrari M. The receptor-mediated endocytosis of nonspherical particles. Biophysical Journal. 2008;94:3790-7.

Decuzzi P, Pasqualini R, Arap W, Ferrari M. Intravascular delivery of particulate systems: Does geometry really matter? Pharmaceutical Research. 2009;26:235-43.

Demchenko AP. Optimization of fluorescence response in the design of molecular biosensors. Analytical biochemistry. 2005;343:1-22.

Demchenko AP. Advanced fluorescent reporters in chemistry and biology II, 2010.

Descalzo AB, Somoza C, Moreno-Bondi MC, Orellana G. Luminescent core-shell imprinted nanoparticles engineered for targeted förster resonance energy transfer-based sensing. Analytical Chemistry. 2013;85:5316-20.

Deshayes S, Maurizot V, Clochard MC, Baudin C, Berthelot T, Esnouf S, et al. "Click" conjugation of peptide on the surface of polymeric nanoparticles for targeting tumor angiogenesis. Pharmaceutical Research. 2011;28:1631-42.

Dey R, Mazumder S, Mitra MK, Mukherjee S, Das GC. Review: Biofunctionalized quantum dots in biology and medicine. Journal of Nanomaterials. 2009, art. no. 815734.

Diltemiz SE, Say R, Buyuktiryaki S, Hur D, Denizli A, Ersoz A. Quantum dot nanocrystals having guanosine imprinted nanoshell for DNA recognition. Talanta. 2008;75:890-6.

Doussineau T, Trupp S, Mohr GJ. Ratiometric pH-nanosensors based on rhodamine-doped silica nanoparticles functionalized with a naphthalimide derivative. Journal of Colloid and Interface Science. 2009;339:266-70.

Duan H, Nie S. Cell-penetrating quantum dots based on multivalent and endosome-disrupting surface coatings. Journal of the American Chemical Society. 2007;129:3333-6.

Evans CE, Lovell PA. Click chemistry as a route to surface functionalization of polymer particles dispersed in aqueous media. Chemical Communications. 2009:2305-7.

Fan C, Wang S, Hong JW, Bazan GC, Plaxco KW, Heeger AJ. Beyond superquenching: Hyperefficient energy transfer from conjugated polymers to gold nanoparticles. Proceedings of the National Academy of Sciences of the United States of America. 2003;100:6297-301.

Ferrari M. Nanogeometry: Beyond drug delivery. Nature Nanotechnology. 2008;3:131-2.

Fraix A, Kandoth N, Manet I, Cardile V, Graziano ACE, Gref R, et al. An engineered nanoplatform for bimodal anticancer phototherapy with dual-color fluorescence detection of sensitizers. Chemical Communications. 2013;49:4459-61.

Frigoli M, Ouadahi K, Larpent C. A cascade FRET-mediated ratiometric sensor for $\mathrm{Cu}^{2+}$ ions based on dual fluorescent ligand-coated polymer nanoparticles. Chemistry - A European Journal. 2009;15:8319-30.

Gai L, Chen H, Zou B, Lu H, Lai G, Li Z, Shen Z. Ratiometric fluorescence chemodosimeters for fluoride anion based on pyrene excimer/monomer transformation. Chemical Communications. 2012;48:10721-3.

Gao D, Zhang, Z., Wu, M., Xie, C., Guan, G., Wang, D. A surface functional monomer-directing strategy for highly dense imprinting of TNT at surface of silica nanoparticles. Journal of the American Chemical Society. 2007;129:7859-66.

Gao F, Luo F, Chen X, Yao W, Yin J, Yao Z, Wang L. A novel nonenzymatic fluorescent sensor for glucose based on silica nanoparticles doped with europium coordination compound. Talanta. 2009;80:202-6. 
Gao F, Luo F, Yin J, Wang L. Preparation of aminated core-shell fluorescent nanoparticles and their application to the synchronous fluorescence determination of $\gamma$-globulin. Luminescence. 2008;23:392-6.

Gao X, Cui Y, Levenson RM, Chung LWK, Nie S. In vivo cancer targeting and imaging with semiconductor quantum dots. Nature biotechnology. 2004;22:969-76.

Ge S, Lu J, Ge L, Yan M, Yu J. Development of a novel deltamethrin sensor based on molecularly imprinted silica nanospheres embedded CdTe quantum dots. Spectrochimica Acta - Part A: Molecular and Biomolecular Spectroscopy. 2011;79:1704-9.

Gota C, Okabe K, Funatsu T, Harada Y, Uchiyama S. Hydrophilic fluorescent nanogel thermometer for intracellular thermometry. Journal of the American Chemical Society. 2009;131:2766-7.

Goutayer M, Dufort S, Josserand V, Royère A, Heinrich E, Vinet F, Bibette J, Coll J L, Texier I. Tumor targeting of functionalized lipid nanoparticles: Assessment by in vivo fluorescence imaging. European Journal of Pharmaceutics and Biopharmaceutics. 2010;75:137-47.

Guan G, Liu B, Wang Z, Zhang Z. Imprinting of Molecular Recognition Sites on Nanostructures and Its Applications in Chemosensors. Sensors. 2008;8:8291-320.

Harrison J, Bartlett CA, Cowin G, Nicholls PK, Evans CW, Clemons TD, Zdyrko B, Luzinov IA Harvey AR, Iyer KS, Dunlop SA, Fitzgerald M. In vivo imaging and biodistribution of multimodal polymeric nanoparticles delivered to the optic nerve. Small. 2012;8:1579-89.

He-Fang Wang YH, Tian-Rong Ji, and Xiu-Ping Yan. Surface Molecular Imprinting on Mn-Doped $\mathrm{ZnS}$ Quantum Dots for Room-Temperature Phosphorescence Optosensing of Pentachlorophenol in Water. Analitical Chemistry. 2009;81: 1615-21.

He C, Yin L, Tang C, Yin C. Size-dependent absorption mechanism of polymeric nanoparticles for oral delivery of protein drugs. Biomaterials. 2012;33:8569-78.

$\mathrm{He} \mathrm{X}$, Nie $\mathrm{H}$, Wang $\mathrm{K}$, Tan $\mathrm{W}$, Wu X, Zhang $\mathrm{P}$. In vivo study of biodistribution and urinary excretion of surface-modified silica nanoparticles. Analytical Chemistry. 2008;80:9597-603.

Hezinger AFE, Teßmar J, Göpferich A. Polymer coating of quantum dots - A powerful tool toward diagnostics and sensorics. European Journal of Pharmaceutics and Biopharmaceutics. 2008;68:13852.

Hong G, Yuan R, Liang B, Shen J, Yang X, Shuai X. Folate-functionalized polymeric micelle as hepatic carcinoma-targeted, MRI-ultrasensitive delivery system of antitumor drugs. Biomedical Microdevices. 2008;10:693-700.

Hong $\mathrm{X}$, Li J, Wang $\mathrm{M}, \mathrm{Xu} \mathrm{J}$, Guo W, Bai Y, Li T. Fabrication of magnetic luminescent nanocomposites by a layer-by-layer self-assembly approach. Chemistry of Materials. 2004;16:40227.

Hong Y, Lam JWY, Tang BZ. Aggregation-induced emission: Phenomenon, mechanism and applications. Chemical Communications. 2009:4332-53.

Hong Y, Lam JWY, Tang BZ. Aggregation-induced emission. Chemical Society Reviews. 2011;40:5361-88.

Hornig S, Biskup C, Gräfe A, Wotschadlo J, Liebert T, Mohr GJ, Heinze T. Biocompatible fluorescent nanoparticles for pH-sensoring. Soft Matter. 2008;4:1169-72.

Hu J, Dai L, Liu S. Analyte-reactive amphiphilic thermoresponsive diblock copolymer micellesbased multifunctional ratiometric fluorescent chemosensors. Macromolecules. 2011a;44:4699-710.

$\mathrm{Hu}$ J, Li C, Cui Y, Liu S. Highly selective colorimetric and fluorometric probes for fluoride ions based on nitrobenzofurazan-containing polymers. Macromolecular Rapid Communications. 2011b;32:610-5.

$\mathrm{Hu}$ J, Liu S. Responsive polymers for detection and sensing applications: Current status and future developments. Macromolecules. 2010;43:8315-30.

Hun X, Zhang Z. Fluoroimmunoassay for tumor necrosis factor- $\alpha$ in human serum using $\mathrm{Ru}$ (bpy) ${ }_{3} \mathrm{Cl}_{2}$-doped fluorescent silica nanoparticles as labels. Talanta. 2007;73:366-71. 
Hwang DW, Ko HY, Lee JH, Kang H, Ryu SH, Song IC, Lee DS, Kim S. A nucleolin-targeted multimodal nanoparticle imaging probe for tracking cancer cells using an aptamer. Journal of Nuclear Medicine. 2010;51:98-105.

Jain KK. Nanotechnology in clinical laboratory diagnostics. Clinica chimica acta; international journal of clinical chemistry. 2005;358:37-54.

Jain KK. Applications of nanobiotechnology in clinical diagnostics. Clinical chemistry. 2007;53:2002-9.

Jana NR, Ying JY. Synthesis of functionalized Au nanoparticles for protein detection. Advanced Materials. 2008;20:430-4.

Jiang Y, Hu X, Hu J, Liu H, Zhong H, Liu S. Reactive fluorescence turn-on probes for fluoride ions in purely aqueous media fabricated from functionalized responsive block copolymers. Macromolecules. 2011;44:8780-90.

Kelly KA, Setlur SR, Ross R, Anbazhagan R, Waterman P, Rubin MA, et al. Detection of early prostate cancer using a hepsin-targeted imaging agent. Cancer Research. 2008;68:2286-91.

Kim J, Kim HS, Lee N, Kim T, Kim H, Yu T, Song IC, Moon WK, Hyeon T. Multifunctional uniform nanoparticles composed of a magnetite nanocrystal core and a mesoporous silica shell for magnetic resonance and fluorescence imaging and for drug delivery. Angewandte Chemie International Edition. 2008;47:8438-41.

Kim Y, Jeon JB, Chang JY. CdSe quantum dot-encapsulated molecularly imprinted mesoporous silica particles for fluorescent sensing of bisphenol A. Journal of Materials Chemistry. 2012;22:24075-80.

Knopp D, Tang D, Niessner R. Review: Bioanalytical applications of biomolecule-functionalized nanometer-sized doped silica particles. Analytica chimica acta. 2009;647:14-30.

Kurkina T, Balasubramanian $\mathrm{K}$. Towards in vitro molecular diagnostics using nanostructures. Cellular and molecular life sciences : CMLS. 2011.

Lee CM, Jeong HJ, Cheong SJ, Kim EM, Kim DW, Lim ST, Sohn MH. Prostate cancer-targeted imaging using magnetofluorescent polymeric nanoparticles functionalized with bombesin. Pharmaceutical Research. 2010a;27:712-21.

Lee D, Erigala VR, Dasari M, Yu J, Dickson RM, Murthy N. Detection of hydrogen peroxide with chemiluminescent micelles. International Journal of Nanomedicine. 2008;3:471-6.

Lee D, Khaja S, Velasquez-Castano JC, Dasari M, Sun C, Petros J, Taylor WR, Murthy N. In vivo imaging of hydrogen peroxide with chemiluminescent nanoparticles. Nature Materials. 2007;6:7659.

Lee I, Hwang O, Yoo D, Khang G, Lee D. Detection of hydrogen peroxide in vitro and in vivo using peroxalate chemiluminescent micelles. Bulletin of the Korean Chemical Society. 2011;32:2187-92.

Lee MH, Chen YC, Ho MH, Lin HY. Optical recognition of salivary proteins by use of molecularly imprinted poly(ethylene-co-vinyl alcohol)/quantum dot composite nanoparticles. Analytical and bioanalytical chemistry. 2010b;397:1457-66.

Lee S, Ryu JH, Park K, Lee A, Lee SY, Youn IC, Ahn CH, Yoon SM, Myung SJ, Moon DH, Chen

X, Choi K, Kwon IC, Kim K. Polymeric nanoparticle-based activatable near-infrared nanosensor for protease determination in vivo. Nano Letters. 2009;9:4412-6.

Lerch S, Dass M, Musyanovych A, Landfester K, Mailänder V. Polymeric nanoparticles of different sizes overcome the cell membrane barrier. European Journal of Pharmaceutics and Biopharmaceutics. 2013;84:265-74.

Lewinski N, Colvin V, Drezek R. Cytotoxicity of nanoparticles. Small. 2008;4:26-49.

Li C, Liu S. Polymeric assemblies and nanoparticles with stimuli-responsive fluorescence emission characteristics. Chemical Communications. 2012;48:3262-78.

Li F, Westphal AH, Marcelis ATM, Sudhölter EJR, Cohen Stuart MA, Leermakers FAM. Thermally sensitive dual fluorescent polymeric micelles for probing cell properties. Soft Matter. 2011;7:11211-5. 
Li H, Li Y, Cheng J. Molecularly imprinted silica nanospheres embedded Cdse quantum dots for highly selective and sensitive optosensing of pyrethroids. Chemistry of Materials. 2010;22:2451-7.

$\mathrm{Li} \mathrm{K}$, Liu B. Water-soluble conjugated polymers as the platform for protein sensors. Polymer Chemistry. 2010;1:252-9.

Li YY, Cheng H, Zhu JL, Yuan L, Dai Y, Cheng SX, Zhang XZ, Zhuo RX. Temperature- and pHsensitive multicolored micellar complexes. Advanced Materials. 2009;21:2402-6.

Liang M, Liu X, Cheng D, Liu G, Dou S, Wang Y, Rusckowski M, Hnatowich DJ. Multimodality nuclear and fluorescence tumor imaging in mice using a streptavidin nanoparticle. Bioconjugate Chemistry. 2010;21:1385-8.

Lin C, Joseph A, Chang C, Lee Y. Molecularly imprinted polymeric film on semiconductor nanoparticles Analyte detection by quantum dot photoluminescence. Journal of Chromatography A. 2004;1027:259-62.

Lin HY, Ho MS, Lee MH. Instant formation of molecularly imprinted poly(ethylene-co-vinyl alcohol)/quantum dot composite nanoparticles and their use in one-pot urinalysis. Biosensors \& bioelectronics. 2009;25:579-86.

Liu A, Wu L, He Z, Zhou J. Development of highly fluorescent silica nanoparticles chemically doped with organic dye for sensitive DNA microarray detection. Analytical and bioanalytical chemistry. 2011a;401:2003-11.

Liu I, Ma C, Zeng F, Wu SZ. Core-shell polymer particles as a fret-based ratiometric sensor for mercury ion detection in water. Acta Polymerica Sinica. 2012:666-72.

Liu R, Guan G, Wang S, Zhang Z. Core-shell nanostructured molecular imprinting fluorescent chemosensor for selective detection of atrazine herbicide. Analyst. 2011b;136:184-90.

Lorenzo RA, Carro AM, Alvarez-Lorenzo C, Concheiro A. To remove or not to remove? The challenge of extracting the template to make the cavities available in molecularly imprinted polymers (MIPs). International Journal of Molecular Sciences. 2011;12:4327-47.

Lu CW, Hung Y, Hsiao JK, Yao M, Chung TH, Lin YS, Wu SH, Hsu SC, Liu HM, Mou CY, Yang

CS, Huang DM, Chen YC. Bifunctional magnetic silica nanoparticles for highly efficient human stem cell labeling. Nano Letters. 2007;7:149-54.

Lu H, Yi G, Zhao S, Chen D, Guo LH, Cheng J. Synthesis and characterization of multi-functional nanoparticles possessing magnetic, up-conversion fluorescence and bio-affinity properties. Journal of Materials Chemistry. 2004;14:1336-41.

Luo J, Xie Z, Lam JWY, Cheng L, Chen H, Qiu C, Hoi Sing K, Zhan X, Liu Y, Zhu D, Ben Zhong T. Aggregation-induced emission of 1-methyl-1,2,3,4,5-pentaphenylsilole. Chemical Communications. 2001:1740-1.

Lutz JF, Zarafshani Z. Efficient construction of therapeutics, bioconjugates, biomaterials and bioactive surfaces using azide-alkyne "click" chemistry. Advanced drug delivery reviews. 2008;60:958-70.

Ma B, Wu S, Zeng F, Luo Y, Zhao J, Tong Z. Nanosized diblock copolymer micelles as a scaffold for constructing a ratiometric fluorescent sensor for metal ion detection in aqueous media. Nanotechnology. 2010;21.

Mayes AG, Whitcombe MJ. Synthetic strategies for the generation of molecularly imprinted organic polymers. Advanced drug delivery reviews. 2005;57:1742-78.

Miki K, Oride K, Inoue S, Kuramochi Y, Nayak RR, Matsuoka H, Harada H, Hiraoka M, Ohe K. Ring-opening metathesis polymerization-based synthesis of polymeric nanoparticles for enhanced tumor imaging in vivo: Synergistic effect of folate-receptor targeting and PEGylation. Biomaterials. 2010;31:934-42.

Mulder WJM, Castermans K, Van Beijnum JR, Oude Egbrink MGA, Chin PTK, Fayad ZA, et al. Molecular imaging of tumor angiogenesis using $\alpha v \beta 3$-integrin targeted multimodal quantum dots. Angiogenesis. 2009;12:17-24.

Nakamura M, Shono M, Ishimura K. Synthesis, characterization, and biological applications of multifluorescent silica nanoparticles. Analytical Chemistry. 2007;79:6507-14. 
Ntziachristos V, Bremer C, Weissleder R. Fluorescence imaging with near-infrared light: New technological advances that enable in vivo molecular imaging. European Radiology. 2003;13:195208.

Owens DE, Peppas NA. Opsonization, biodistribution, and pharmacokinetics of polymeric nanoparticles. Int J Pharm. 2006;307:93-102.

Park JH, Gu L, Von Maltzahn G, Ruoslahti E, Bhatia SN, Sailor MJ. Biodegradable luminescent porous silicon nanoparticles for in vivo applications. Nature Materials. 2009;8:331-6.

Peng HS, Stolwijk JA, Sun LN, Wegener J, Wolfbeis OS. A nanogel for ratiometric fluorescent sensing of intracellular pH values. Angewandte Chemie - International Edition. 2010;49:4246-9.

Piletska EV, Guerreiro AR, Whitcombe MJ, Piletsky SA. Influence of the polymerization conditions on the performance of molecularly imprinted polymers. Macromolecules. 2009;42:4921 8 .

Piletsky SA, Turner NW, Laitenberger P. Molecularly imprinted polymers in clinical diagnostics-future potential and existing problems. Medical engineering \& physics. 2006;28:971-7.

Poma A, Turner AP, Piletsky SA. Advances in the manufacture of MIP nanoparticles. Trends in biotechnology. 2010;28:629-37.

Pucci A, Locatelli E, Ponti J, Uboldi C, Molinari V, Comes Franchini M. Click chemistry on the surface of PLGA-b-PEG polymeric nanoparticles: a novel targetable fluorescent imaging nanocarrier. Journal of Nanoparticle Research. 2013;15:1-6.

Qian K, Fang G, Wang S. Highly sensitive and selective novel core-shell molecularly imprinted polymer based on NaYF4: $\mathrm{Yb3}+$, Er3+ upconversion fluorescent nanorods. RSC Advances. 2013;3:3825-8.

Rao JP, Geckeler KE. Polymer nanoparticles: Preparation techniques and size-control parameters. Progress in Polymer Science (Oxford). 2011;36:887-913.

Renub-Research. Global In Vitro Diagnostic (IVD) Market Trends and Future Forecast (20092014), 2010.

Riehemann K, Schneider SW, Luger TA, Godin B, Ferrari M, Fuchs H. Nanomedicine - Challenge and perspectives. Angewandte Chemie - International Edition. 2009;48:872-97.

RNCOS. Global in vitro diagnostic market analysis2011.

Rowe MD, Tham DH, Kraft SL, Boyes SG. Polymer-modified gadolinium metal-organic framework nanoparticles used as multifunctional nanomedicines for the targeted imaging and treatment of cancer. Biomacromolecules. 2009;10:983-93.

Sahoo SK, Parveen S, Panda JJ. The present and future of nanotechnology in human health care. Nanomedicine : nanotechnology, biology, and medicine. 2007;3:20-31.

Salgueiriño-Maceira V, Correa-Duarte MA, Spasova M, Liz-Marzán LM, Farle M. Composite silica spheres with magnetic and luminescent functionalities. Advanced Functional Materials. 2006;16:509-14.

Selvan ST, Patra PK, Ang CY, Ying JY. Synthesis of silica-coated semiconductor and magnetic quantum dots and their use in the imaging of live cells. Angewandte Chemie - International Edition. 2007;46:2448-52.

Selvan ST, Yang Tan TT, Kee Yi D, Jana NR. Functional and multifunctional nanoparticles for bioimaging and biosensing. Langmuir. 2010;26:11631-41.

Sheng Y, Liu C, Yuan Y, Tao X, Yang F, Shan X, Zhou H, Xu F. Long-circulating polymeric nanoparticles bearing a combinatorial coating of PEG and water-soluble chitosan. Biomaterials. 2009;30:2340-8.

Shi H, He X, Yuan Y, Wang K, Liu D. Nanoparticle-based biocompatible and long-life marker for lysosome labeling and tracking. Analytical Chemistry. 2010;82:2213-20.

Shiraishi Y, Inoue T, Hirai T. Local viscosity analysis of triblock copolymer micelle with cyanine dyes as a fluorescent probe. Langmuir. 2010;26:17505-12.

Song Y, Wei W, Qu X. Colorimetric biosensing using smart materials. Adv Mater. 2011;23:421536. 
Sperling RA, Parak WJ. Surface modification, functionalization and bioconjugation of colloidal Inorganic nanoparticles. Philosophical Transactions of the Royal Society A: Mathematical, Physical and Engineering Sciences. 2010;368:1333-83.

Stark WJ. Nanoparticles in Biological Systems. Angewandte Chemie International Edition. 2011;50:1242-58.

Su H, Liu Y, Wang D, Wu C, Xia C, Gong Q, Song B, Ai H. Amphiphilic starlike dextran wrapped superparamagnetic iron oxide nanoparticle clsuters as effective magnetic resonance imaging probes. Biomaterials. 2013;34:1193-203.

Sudimack J, Lee RJ. Targeted drug delivery via the folate receptor. Advanced drug delivery reviews. 2000;41:147-62.

Suen WLL, Chau Y. Specific uptake of folate-decorated triamcinolone-encapsulating nanoparticles by retinal pigment epithelium cells enhances and prolongs antiangiogenic activity. Journal of Controlled Release. 2013;167:21-8.

Svenson J, Nicholls IA. On the thermal and chemical stability of molecularly imprinted polymers. Analytica chimica acta. 2001;435:19-24.

Tang L, Jin JK, Qin A, Zhang Yuan W, Mao Y, Mei J, Zhi Sun J, Zhong Tang B. A fluorescent thermometer operating in aggregation-induced emission mechanism: Probing thermal transitions of PNIPAM in water. Chemical Communications. 2009:4974-6.

Tian J, Chen H, Zhuo L, Xie Y, Li N, Tang B. A highly selective, cell-permeable fluorescent nanoprobe for ratiometric detection and imaging of peroxynitrite in living cells. Chemistry - A European Journal. 2011;17:6626-34.

Tissandier C, Diop N, Martini M, Roux S, Tillement O, Hamaide T. One-pot synthesis of hybrid multifunctional silica nanoparticles with tunable coating by click chemistry in reverse W/O microemulsion. Langmuir. 2012;28:209-18.

Tokonami S, Shiigi H, Nagaoka T. Review: Micro- and nanosized molecularly imprinted polymers for high-throughput analytical applications. Analytica chimica acta. 2009;641:7-13.

Tsai HY, Chang CY, Li YC, Chu WC, Viswanathan K, Bor Fuh C. Detection of carcinoembryonic antigen using functional magnetic and fluorescent nanoparticles in magnetic separators. Journal of Nanoparticle Research. 2011;13:2461-7.

Tsien RY. A non-disruptive technique for loading calcium buffers and indicators into cells. Nature. 1981;290:527-8.

Uchiyama S, Kawai N, De Silva AP, Iwai K. Fluorescent Polymeric AND Logic Gate with Temperature and $\mathrm{pH}$ as Inputs. Journal of the American Chemical Society. 2004;126:3032-3.

Uchiyama S, Matsumura Y, De Silva AP, Iwai K. Fluorescent molecular thermometers based on polymers showing temperature-induced phase transitions and labeled with polarity-responsive benzofurazans. Analytical Chemistry. 2003;75:5926-35.

Vollrath A, Schubert S, Schubert US. Fluorescence imaging of cancer tissue based on metal-free polymeric nanoparticles-a review. Journal of Materials Chemistry B. 2013;1:1994-2007.

Wan X, Liu T, Liu S. Thermoresponsive core cross-linked micelles for selective ratiometric fluorescent detection of $\mathrm{Hg}^{2+}$ ions. Langmuir. 2011;27:4082-90.

Wang D, Miyamoto R, Shiraishi Y, Hirai T. BODIPY-conjugated thermoresponsive copolymer as a fluorescent thermometer based on polymer microviscosity. Langmuir. 2009;25:13176-82.

Wang L, Tan W. Multicolor FRET silica nanoparticles by single wavelength excitation. Nano Letters. 2006;6:84-8.

Wang L, Wang, K., Santra, S., Zhao, X., Hilliard, L.R., Smith, J.E., Wu, Y., Tan, W. Watching Silica nanoparticles glow in the biological world. Analytical Chemistry. 2006; 78:646-54.

Wang Y, Li Z, Zhong W, Li H, Xu D, Chen H. Rhodamine B doped silica nanoparticle labels for protein microarray detection. Science China Chemistry. 2010a;53:747-51.

Wang Y, $\mathrm{Pu} \mathrm{KY,} \mathrm{Liu} \mathrm{B.} \mathrm{Anionic} \mathrm{conjugated} \mathrm{polymer} \mathrm{with} \mathrm{aptamer-functionalized} \mathrm{silica}$ nanoparticle for label-free naked-eye detection of lysozyme in protein mixtures. Langmuir. 2010b;26:10025-30. 
Weng J, Ren J. Luminescent quantum dots: A very attractive and promising tool in biomedicine. Current Medicinal Chemistry. 2006;13:897-909.

Wong YJ, Zhu L, Teo WS, Tan YW, Yang Y, Wang C, Chen H. Revisiting the Stöber method: Inhomogeneity in silica shells. Journal of the American Chemical Society. 2011;133:11422-5.

Wu H, Huo Q, Varnum S, Wang J, Liu G, Nie Z, Liu J, Lin Y. Dye-doped silica nanoparticle labels/protein microarray for detection of protein biomarkers. Analyst. 2008;133:1550-5.

Wu S, Han G, Milliron DJ, Aloni S, Altoe V, Talapin DV, Cohen BE, Schuck PJ. Non-blinking and photostable upconverted luminescence from single lanthanide-doped nanocrystals. Proceedings of the National Academy of Sciences of the United States of America. 2009a;106:10917-21.

$\mathrm{Wu} \mathrm{T}$, Zou G, Hu J, Liu S. Fabrication of photoswitchable and thermotunable multicolor fluorescent hybrid silica nanoparticles coated with dye-labeled poly $(\mathrm{N}$-isopropylacrylamide) brushes. Chemistry of Materials. 2009b;21:3788-98.

Wu W, Shen J, Li Y, Zhu H, Banerjee P, Zhou S. Specific glucose-to-SPR signal transduction at physiological $\mathrm{pH}$ by molecularly imprinted responsive hybrid microgels. Biomaterials. 2012;33:7115-25.

Xing Y, Xia Z, Rao J. Semiconductor quantum dots for biosensing and in vivo imaging. IEEE Transactions on Nanobioscience. 2009;8:4-12.

$\mathrm{Xu}$ J, Sun L, Li J, Liang J, Zhang H, Yang W. Fitc and Ru(phen) ${ }_{3}{ }^{2+}$ co-doped silica particles as visualized ratiometric $\mathrm{pH}$ indicator. Nanoscale Research Letters. 2011; 6:1-7.

Yang K, Berg MM, Zhao C, Ye L. One-pot synthesis of hydrophilic molecularly imprinted nanoparticles. Macromolecules. 2009a;42:8739-46.

Yang Y.K., Yook K.J., Tae J. A Rhodamine-Based Fluorescent and Colorimetric Chemodosimeter for the Rapid Detection of $\mathrm{Hg}^{2+}$ Ions in Aqueous Media. J Am Chem Soc. 2005; 127:16760-1.

Yang Z, Leon J, Martin M, Harder JW, Zhang R, Liang D, Lu W, Tian M, Gelovani JG, Qiao A, Li

C. Pharmacokinetics and biodistribution of near-infrared fluorescence polymeric nanoparticles. Nanotechnology. 2009b; 20.

Yang Z, Zheng S, Harrison WJ, Harder J, Wen X, Gelovani JG, Qiao A, Li C. Long-circulating near-infrared fluorescence core-cross-linked polymeric micelles: Synthesis, characterization, and dual nuclear/optical imaging. Biomacromolecules. 2007;8:3422-8.

Ye L, Haupt K. Molecularly imprinted polymers as antibody and receptor mimics for assays, sensors and drug discovery. Analytical and bioanalytical chemistry. 2004;378:1887-97.

Yildirimer L, Thanh NTK, Loizidou M, Seifalian AM. Toxicological considerations of clinically applicable nanoparticles. Nano Today. 2011;6:585-607.

Yoo MK, Kim IY, Kim EM, Jeong HJ, Lee CM, Jeong YY, Akaike T, Cho CS. Superparamagnetic iron oxide nanoparticles coated with galactose-carrying polymer for hepatocyte targeting. Journal of Biomedicine and Biotechnology. 2007;2007.

Yoo MK, Park IK, Lim HT, Lee SJ, Jiang HL, Kim YK, Choi YJ, Cho MH, Cho CS. Folate-PEGsuperparamagnetic iron oxide nanoparticles for lung cancer imaging. Acta Biomaterialia. 2012;8:3005-13.

Yoon TJ, Kim JS, Kim BG, Yu KN, Cho MH, Lee JK. Multifunctional nanoparticles possessing a "magnetic motor effect" for drug or gene delivery. Angewandte Chemie - International Edition. 2005;44:1068-71.

You CC, Miranda OR, Gider B, Ghosh PS, Kim IB, Erdogan B, Krovi SA, Bunz UHF, Rotello VM. Detection and identification of proteins using nanoparticle-fluorescent polymer 'chemical nose' sensors. Nature Nanotechnology. 2007;2:318-23.

Yu T, Greish K, McGill LD, Ray A, Ghandehari H. Influence of geometry, porosity, and surface characteristics of silica nanoparticles on acute toxicity: Their vasculature effect and tolerance threshold. ACS Nano. 2012;6:2289-301.

Zetterlund PB, Kagawa Y, Okubo M. Controlled/living radical polymerization in dispersed systems. Chemical Reviews. 2008;108:3747-94. 
Zhang L, Xue H, Gao C, Carr L, Wang J, Chu B, Jiang S. Imaging and cell targeting characteristics of magnetic nanoparticles modified by a functionalizable zwitterionic polymer with adhesive 3,4dihydroxyphenyl-l-alanine linkages. Biomaterials. 2010;31:6582-8.

Zhang R, Xiong C, Huang M, Zhou M, Huang Q, Wen X, Liang D, Li C. Peptide-conjugated polymeric micellar nanoparticles for Dual SPECT and optical imaging of EphB4 receptors in prostate cancer xenografts. Biomaterials. 2011;32:5872-9.

Zhao X, Tapec-Dytioco R, Tan W. Ultrasensitive DNA detection using highly fluorescent bioconjugated nanoparticles. Journal of the American Chemical Society. 2003;125:11474-5.

Zheng C, Zheng M, Gong P, Jia D, Zhang P, Shi B, Sheng Z, Ma Y, Cai L. Indocyanine greenloaded biodegradable tumor targeting nanoprobes for in vitro and in vivo imaging. Biomaterials. 2012;33:5603-9.

Zhou K, Wang Y, Huang X, Luby-Phelps K, Sumer BD, Gao J. Tunable, ultrasensitive pHresponsive nanoparticles targeting specific endocytic organelles in living cells. Angewandte Chemie - International Edition. 2011;50:6109-14.

Zhou XZaJ. Improving the signal sensitivity and photostability of DNA hybridizations. Anal Chem 2004. 2004; 76:5302-12.

Zhu L, Wu W, Zhu MQ, Han JJ, Hurst JK, Li ADQ. Reversibly photoswitchable dual-color fluorescent nanoparticles as new tools for live-cell imaging. Journal of the American Chemical Society. 2007; 129:3524-6.

Zhu MQ, Zhang GF, Li C, Aldred MP, Chang E, Drezek RA, Li ADQ. Reversible two-photon photoswitching and two-photon imaging of immunofunctionalized nanoparticles targeted to cancer cells. Journal of the American Chemical Society. 2011;133:365-72.

Zhu MQ, Zhu L, Han JJ, Wu W, Hurst JK, Li ADQ. Spiropyran-based photochromic polymer nanoparticles with optically switchable luminescence. Journal of the American Chemical Society. 2006;128:4303-9. 


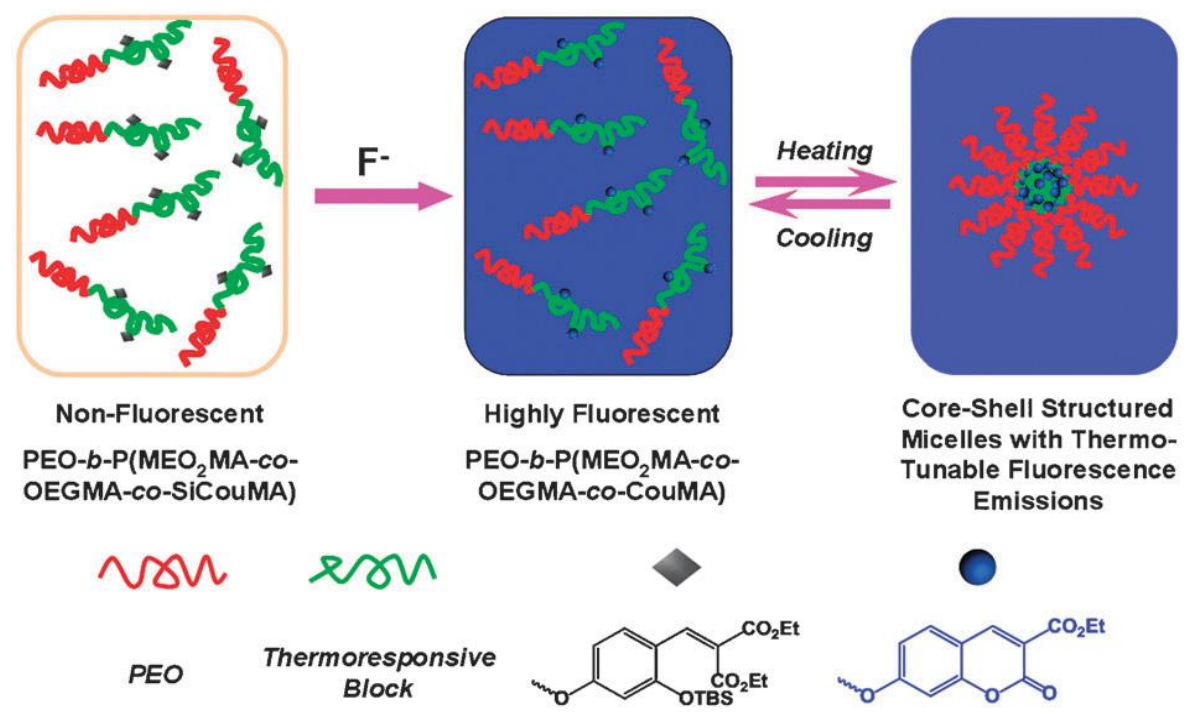

Figure 1. Illustration for the generation of responsive NPs for fluoride ions sensing. The system exploits fluoride ions-induced cyclization reaction of non-fluorescent portions to form fluorescent coumarin moieties within the polymer, where $\mathrm{PEO}, \mathrm{MEO}_{2} \mathrm{MA}$, and OEGMA are poly(ethylene glycol), di(ethylene glycol) monomethyl ether methacrylate, and oligo(ethylene glycol) monomethyl ether methacrylate, respectively. Reproduced with permission from Jiang Y. et al., Macromolecules 2011, 44, 8780. Copyright (2011) American Chemical Society. 

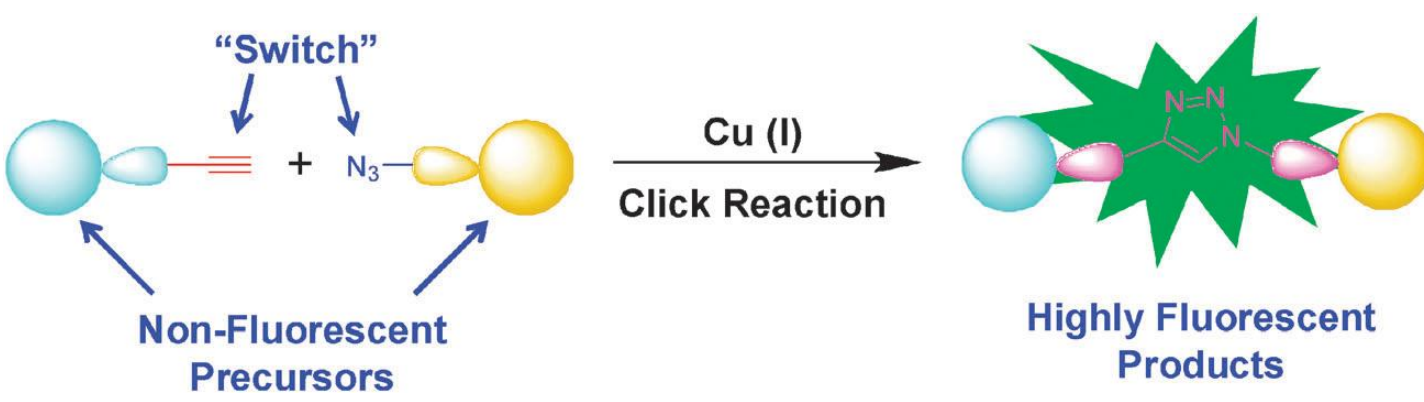

Figure 2. Representation of a typical fluorogenic click reaction. Reproduced from Li, C., Liu, S. Chemical Communications 2012, 48, with permission of The Royal Society of Chemistry. 

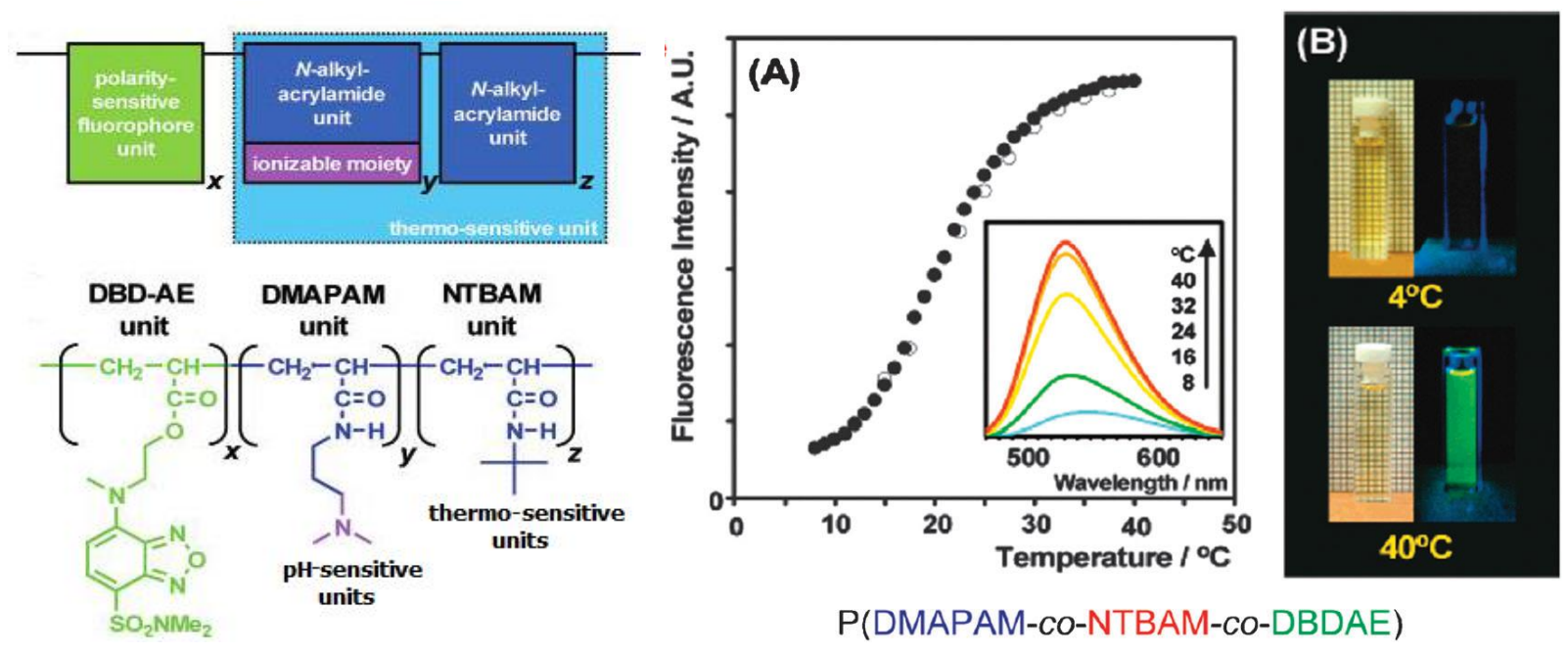

Figure 3. Thermo-responsive copolymer, composed of N,N-dimethylaminopropylacrylamide (DMAPAM), N-t-butylacrylamide (NTBAM) and 4-N-(2-acryloyloxyethyl)-N-methylamino-7-N,N-dimethylaminosulfonyl-2,1,3-benzoxadiazole (DBD-AE), in buffer solution acts as a fluorescent molecular thermometer. (A) Fluorescence intensity changes upon heating and cooling cycles. Inset: fluorescence emission spectra. (B) Visible and fluorescent images of the sample. Adapted with permission from Uchiyama S. et al., Journal of the American Chemical Society 2004, 126, 3032. Copyright (2004) American Chemical Society. 


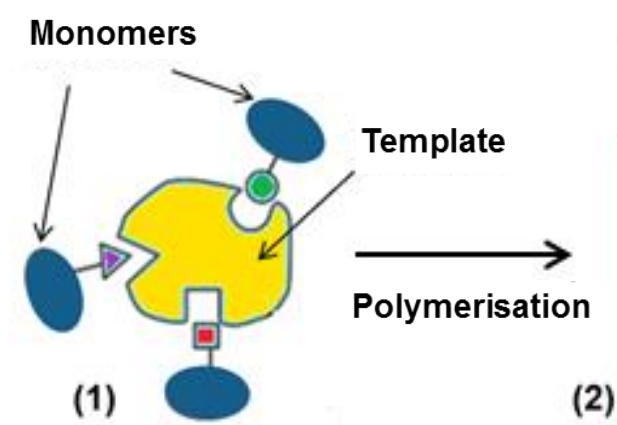

Formation of the prepolymerisation complex

(2)
Polymeric film
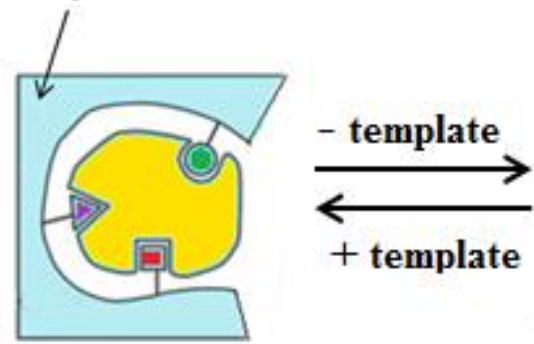

(3)

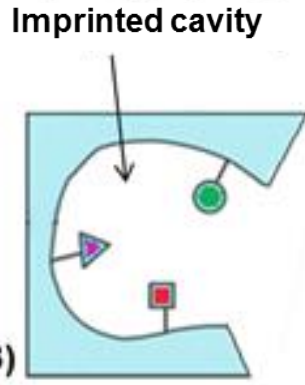

MIP matrix

Figure 4. Schematic diagram of a general MIP process: the molecule to be imprinted (template) is mixed with functional monomers and a cross-linker (here not shown) leading to the formation of a self-assembling prepolymerisation complex (1). The following polymerisation produces a polymeric matrix bearing imprinted sites (2). The eventual removal of the template molecule leaves behind cavities capable of specifically rebind the target molecule (3). 


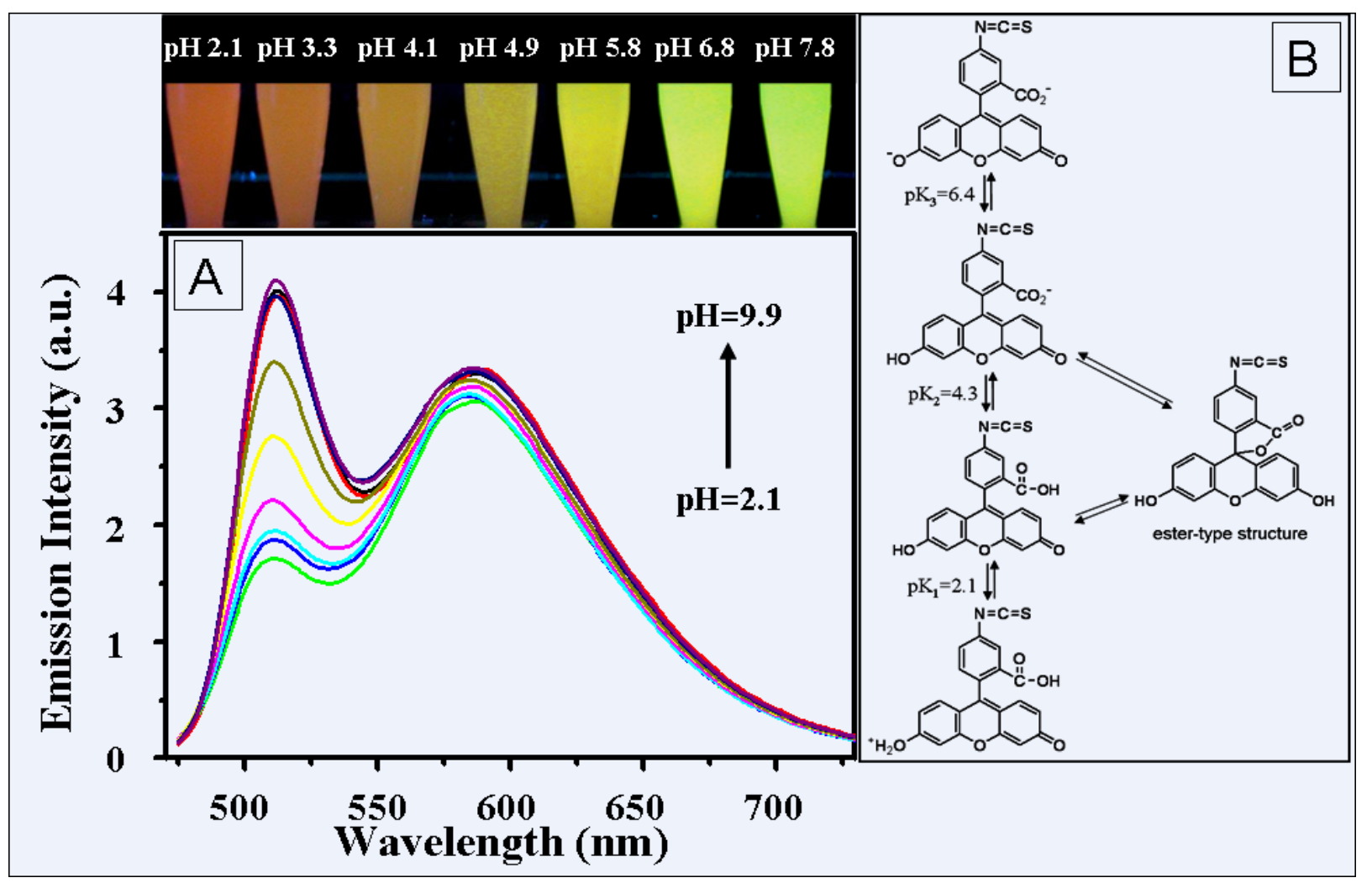

Figure 5. (A) Emission spectra of co-doped SNPs and (B) FITC molecular structures in different pH conditions. Insert contains the images of SNPs dispersed in Britton-Robinson buffer solutions at different $\mathrm{pH}$, under a $365 \mathrm{~nm}$ UV lamp. Reproduced with permission from Xu J. et al., Nanoscale Research Letters 2011, 6, 1. Copyright (2011) Springer. 


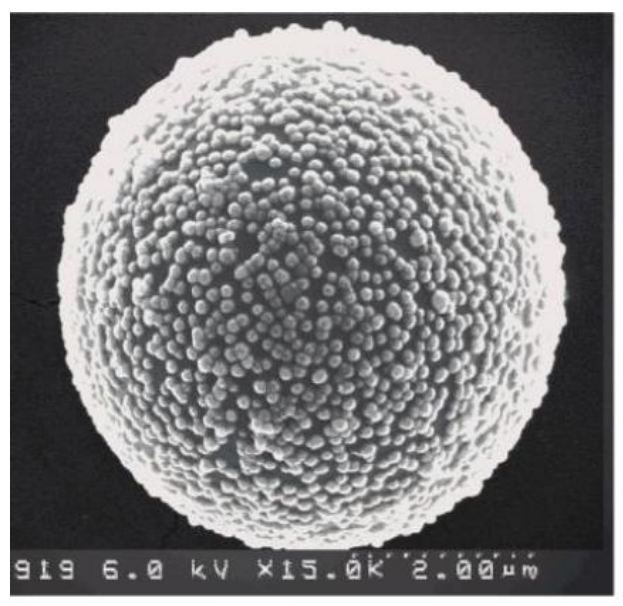

a

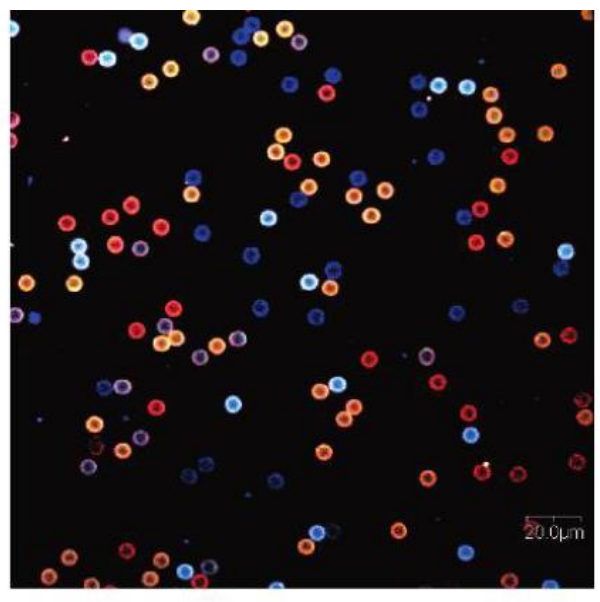

b

Figure 6. (a) SEM image of one microsphere-NP complex (b) Confocal fluorescence image of a mixture of five types of microsphere-NP complexes under 488-nm Argon-ion laser excitation. Reprinted with permission from Wang L.; Tan W., Nano Letters 2006, 6, 84. Copyright (2006) American Chemical Society. 


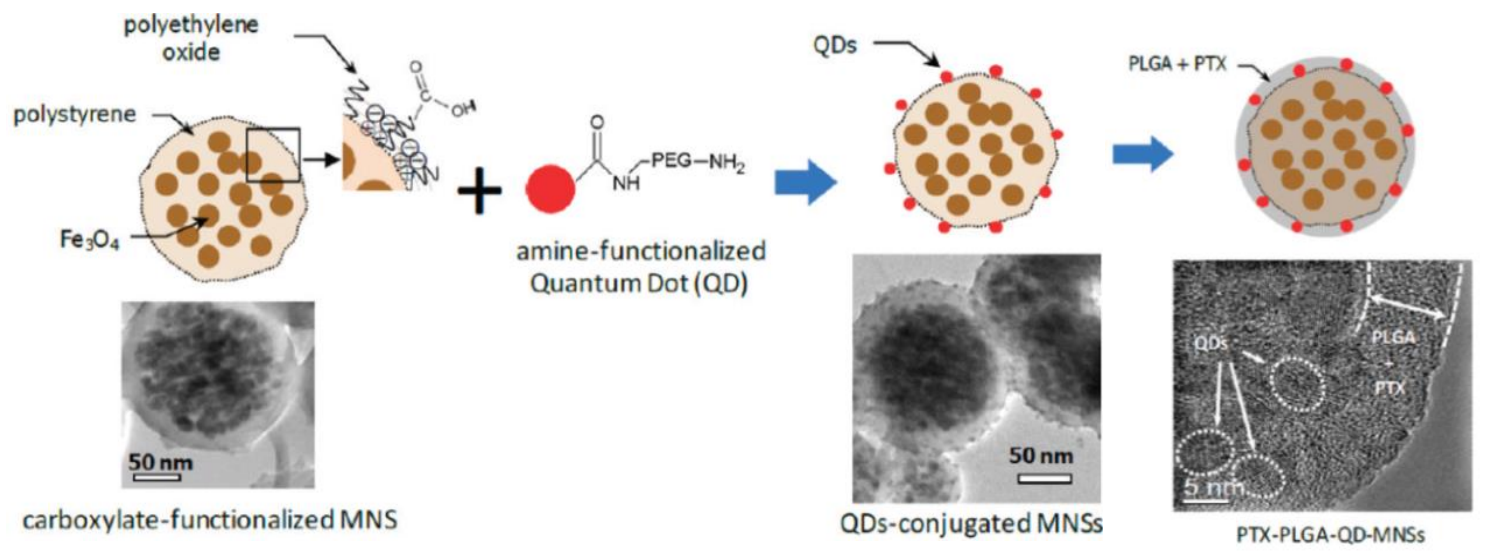

Figure 7. (a) $\mathrm{Fe}_{3} \mathrm{O}_{4}$ nanoparticles are embedded within a polystyrene matrix, which was functionalized for further covalent linkage of PEG-functionalized QDs. Afterwards, the system was coated with poly(lactic-coglycolic acid) (PLGA) layer. Adapted with permission from Cho H. et al., ACS Nano 2010, 4, 5398. Copyright (2010) American Chemical Society. 


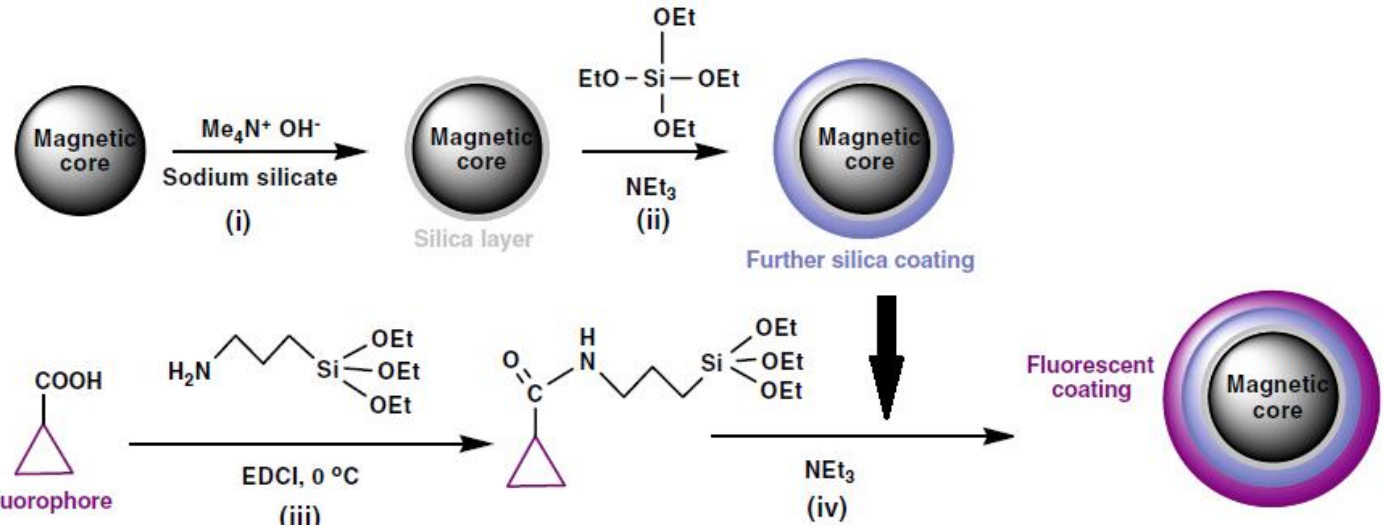

Figure 8. Synthesis of fluorescent silica-coated magnetic NPs. Adapted from Corr S. A. et al., Nanoscale Research Letters 2008, 3, 87. Copyright (2008) Springer. 

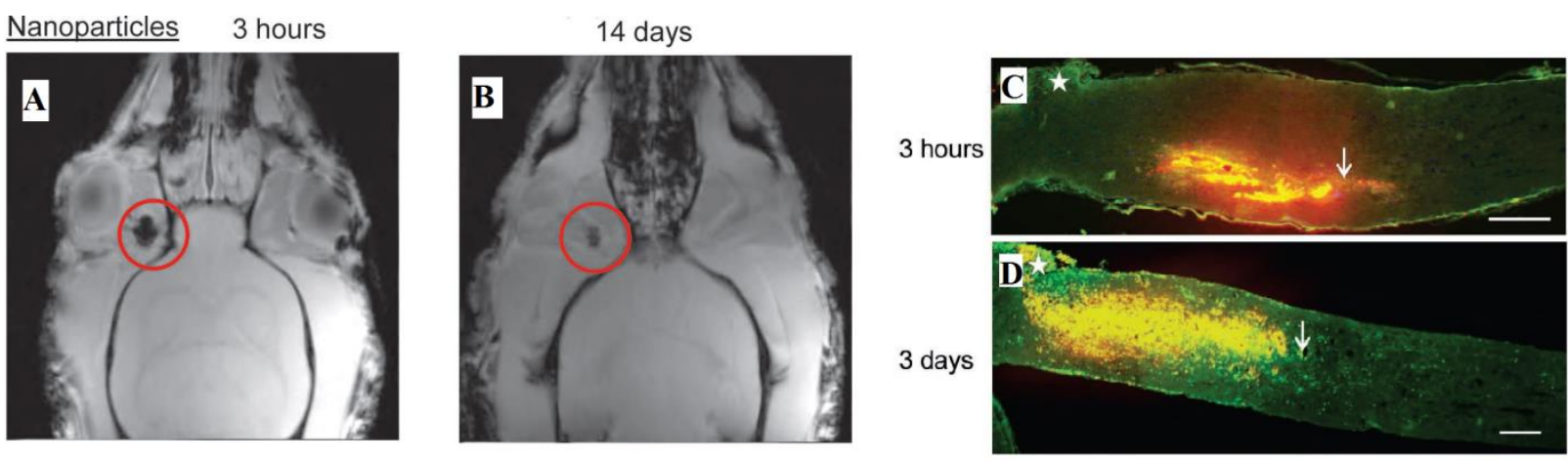

Figure 9. MR images of a rat's head after injection with $0.5 \mu \mathrm{L}$ of multifunctional NPs, after (a) $3 \mathrm{~h}$ and (b) 14 days. Fluorescence microscopy images of optical nerve sections from rats injected with NP (orange area) into the optical nerve injury site, after (c) 3 hours and (d) 3 days. The arrow represents injection site and the injury is indicated by *. Adapted with permission from Harrison J. et al., Small 2012, 8, 1579. Copyright (2012) Wiley. 


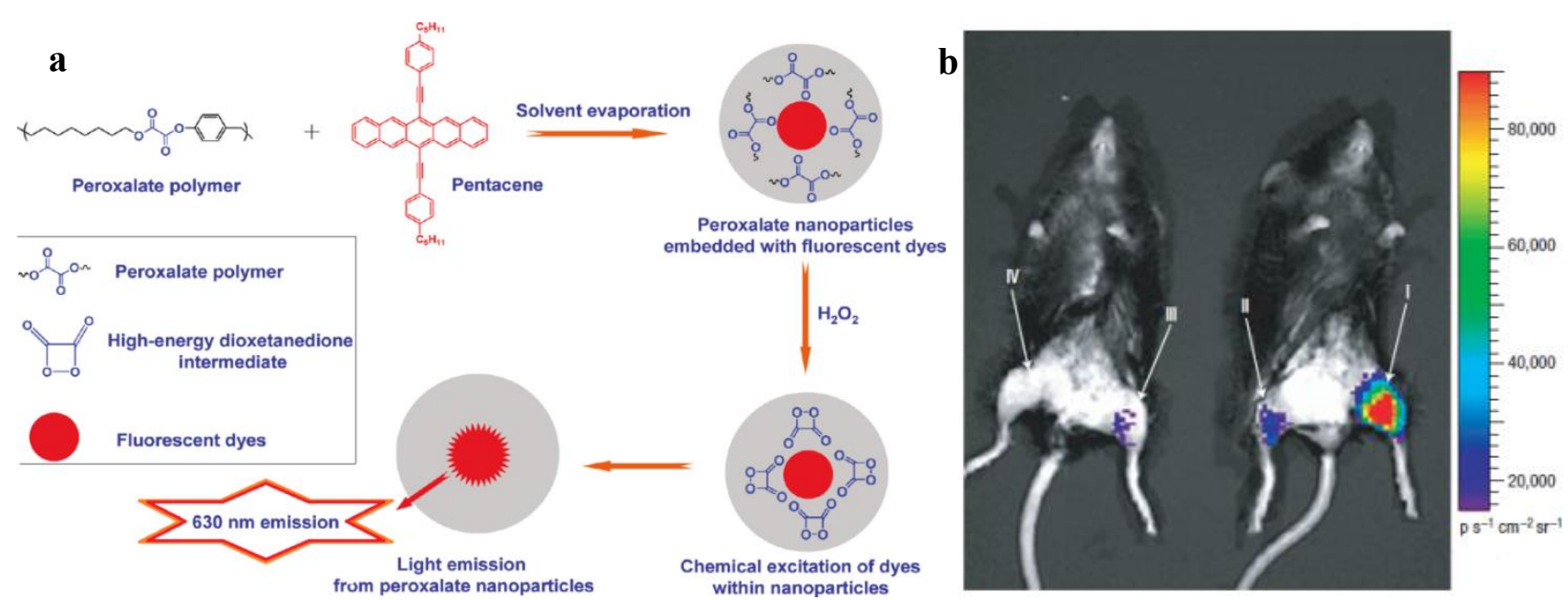

Figure 10. (a) Peroxalate nanoparticles, embedded with pentacene as the fluorescent dye, employed for in vivo $\mathrm{H}_{2} \mathrm{O}_{2}$ imaging through a chemiluminescent process. Adapted with permission from $\mathrm{Hu} \mathrm{J}$; Liu S., Macromolecules 2010, 43, 8315. Copyright (2010) American Chemical Society. (b) In vivo $\mathrm{H}_{2} \mathrm{O}_{2}$ imaging using peroxalate NPs. (I) Peroxalate NPs $+10 \mu \mathrm{M}$ of hydrogen peroxide; (II) peroxalate NPs $+1 \mu \mathrm{M}$ of hydrogen peroxide; (III) peroxalate NPs only; (IV) negative control. Adapted with permission from Lee D. et al., Nature Materials 2007, 6, 765. Copyright (2007) American Chemical Society. 

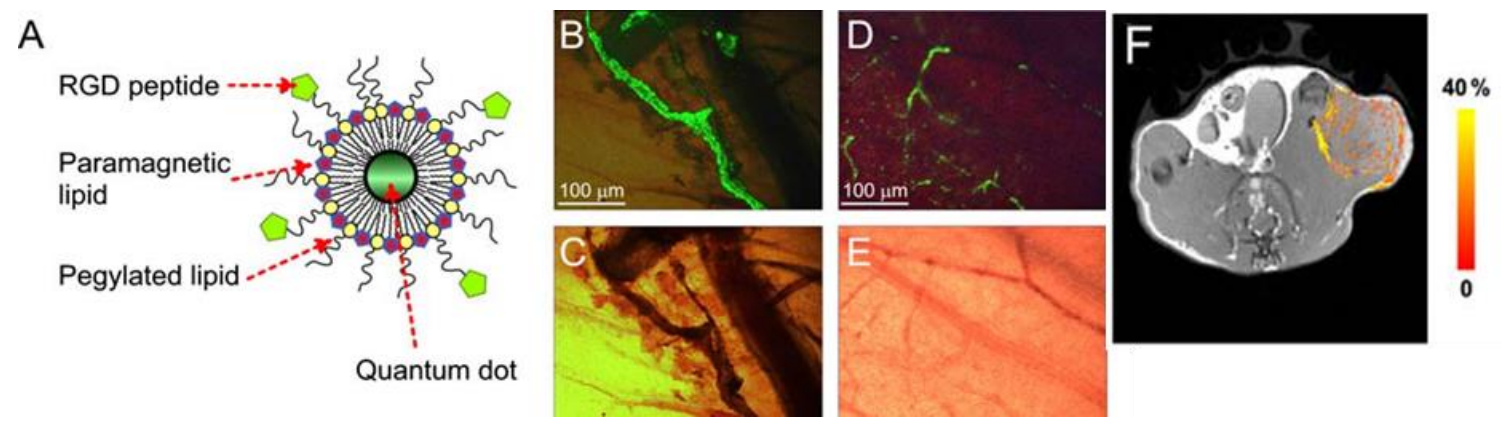

Figure 11. (a) Polymer-paramagnetic QDs for angiogenesis imaging. (b,d) Fluorescence images and (c,e) corresponding bright field images of chorioallantoic membrane with topically growing LS174T human colon carcinoma tissue. (f) MR imaging of the tumor region in mice. Adapted from Mulder W. J. M. et al., Angiogenesis 2009, 12, 17. Copyright (2009) Springer. 


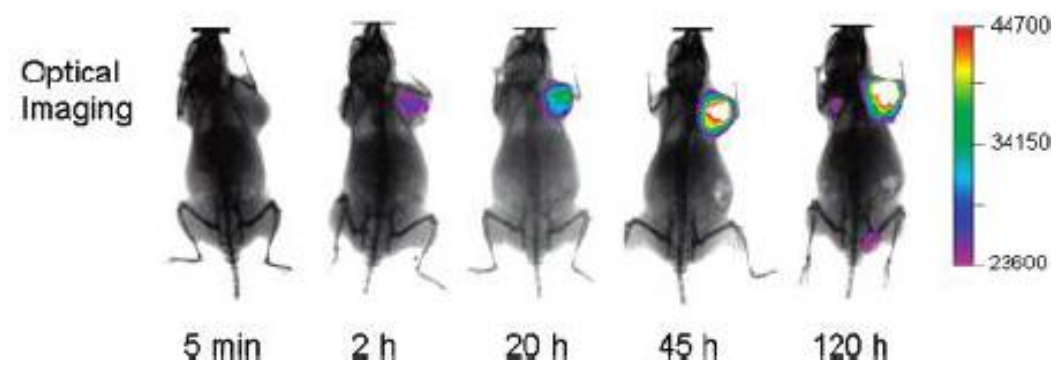

Figure 12. NIRF optical images of mice at different times after injection with PEG-coated micelles (the signal intensity in scale bar is in arbitrary units). Adapted with permission from Yang Z. et al., Biomacromolecules 2007, 8, 3422. Copyright (2007) American Chemical Society. 


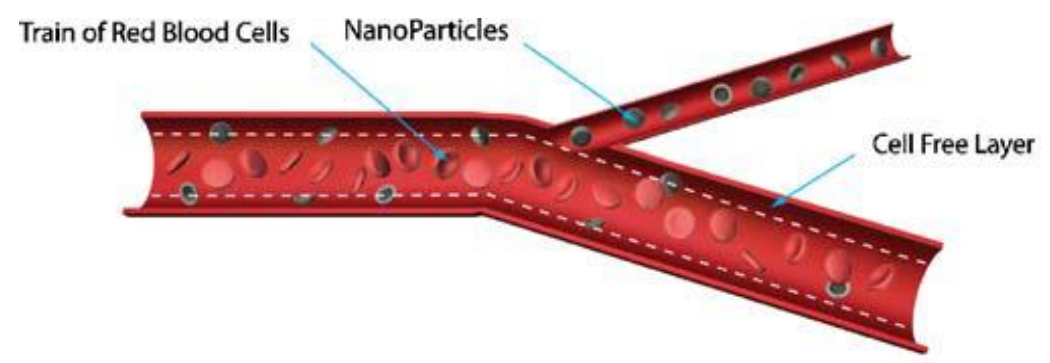

Figure 13. Particles tend to accumulate in proximity to the vessel walls and leave large vessels in favor of smaller ones. Reproduced with permission from Decuzzi P. et al., Pharmaceutical Research 2009, 26, 235. Copyright (2009) Springer. 
Table 1. Comparison of the most frequently employed methods for the synthesis of NP for biomedical application

\begin{tabular}{|llll|}
\hline \multicolumn{4}{c|}{ Organic nanoparticles } \\
\hline Synthetic method & Particle size/initiator & Advantages & Drawbacks/issues \\
\hline $\begin{array}{l}\text { Mini- and micro- } \\
\text { emulsion } \\
\text { polymerization }\end{array}$ & $\begin{array}{l}10-200 \mathrm{~nm} \\
\text { Initiator: persulfate; } \\
\text { azoisobutyronitrile }\end{array}$ & $\begin{array}{l}\text { Performed both in water } \\
\text { and organics. Good } \\
\text { monodispersity }\end{array}$ & $\begin{array}{l}\text { Size control. Surfactant } \\
\text { needed to obtain smaller } \\
\text { particles }\end{array}$ \\
\hline $\begin{array}{l}\text { Living radical } \\
\text { polymerization }\end{array}$ & $\begin{array}{l}30-300 \mathrm{~nm} \\
\text { Initiator: alkoxyamines, } \\
\text { thioesters, alkyl halides, },\end{array}$ & $\begin{array}{l}\text { Performed both in water } \\
\text { and organics. Ideal for } \\
\text { synthesis of functionalised } \\
\text { nanoparticles }\end{array}$ & $\begin{array}{l}\text { Residues of initiator and } \\
\text { surfactant (if employed). }\end{array}$ \\
& Colloidal stability \\
\hline $\begin{array}{l}\text { Precipitation and } \\
\text { dispersion } \\
\text { polymerization }\end{array}$ & $\begin{array}{l}200 \mathrm{~nm}-10 \mu \mathrm{m} \\
\text { Initiator: persulfate; } \\
\text { azoisobutyronitrile }\end{array}$ & $\begin{array}{l}\text { With/without surfactant. } \\
\text { Moderate-good } \\
\text { monodispersity }\end{array}$ & $\begin{array}{l}\text { Size control. Possible } \\
\text { irregular shape. Difficult to } \\
\text { obtain particles<200 nm }\end{array}$ \\
\hline & & Inorganic nanoparticles & \\
\hline Synthetic method & Particle size/catalysis & Advantages & Drawbacks/issues \\
\hline $\begin{array}{l}\text { Microemulsion } \\
\text { polymerization }\end{array}$ & $\begin{array}{l}10-70 \mathrm{~nm} \\
\text { Basic catalysis }\end{array}$ & Good monodispersity & $\begin{array}{l}\text { Presence of surfactant. } \\
\text { Performed in organics }\end{array}$ \\
\hline Sol-gel method & $50 \mathrm{~nm}-2 \mu \mathrm{m}$ & Good monodispersity & Presence of surfactant \\
& Acid/basic catalysis & & Surfactant-free. \\
\hline Stöber method & $\begin{array}{l}15 \mathrm{~nm}-2 \mu \mathrm{m} \\
\text { Basic catalysis }\end{array}$ & Excellent monodispersity & $\begin{array}{l}\text { Difficult to obtain particles } \\
<0 \text { nm }\end{array}$ \\
\hline
\end{tabular}

